\title{
Saturation process induced by vortex-merging in numerical Vlasov-Maxwell experiments of stimulated Raman backscattering
}

M. Albrecht-Marc, A. Ghizzo, T. W. Johnston, T. Réveillé, D. Del Sarto, and P. Bertrand

Citation: Physics of Plasmas 14, 072704 (2007); doi: 10.1063/1.2749715

View online: https://doi.org/10.1063/1.2749715

View Table of Contents: http://aip.scitation.org/toc/php/14/7

Published by the American Institute of Physics

\section{Articles you may be interested in}

Evolution of the stimulated Raman scattering instability in two-dimensional particle-in-cell simulations Physics of Plasmas 17, 092704 (2010); 10.1063/1.3474619

Multidimensional electron beam-plasma instabilities in the relativistic regime

Physics of Plasmas 17, 120501 (2010); 10.1063/1.3514586

Nonlinear plasma response to a slowly varying electrostatic wave, and application to stimulated Raman scattering

Physics of Plasmas 14, 042304 (2007); 10.1063/1.2711819

Parametric instabilities of electromagnetic waves in plasmas

The Physics of Fluids 17, 778 (1974); 10.1063/1.1694789

Electron and ion kinetic effects on non-linearly driven electron plasma and ion acoustic waves

Physics of Plasmas 20, 032107 (2013); 10.1063/1.4794346

Persistent subplasma-frequency kinetic electrostatic electron nonlinear waves

Physics of Plasmas 16, 042105 (2009); 10.1063/1.3094061

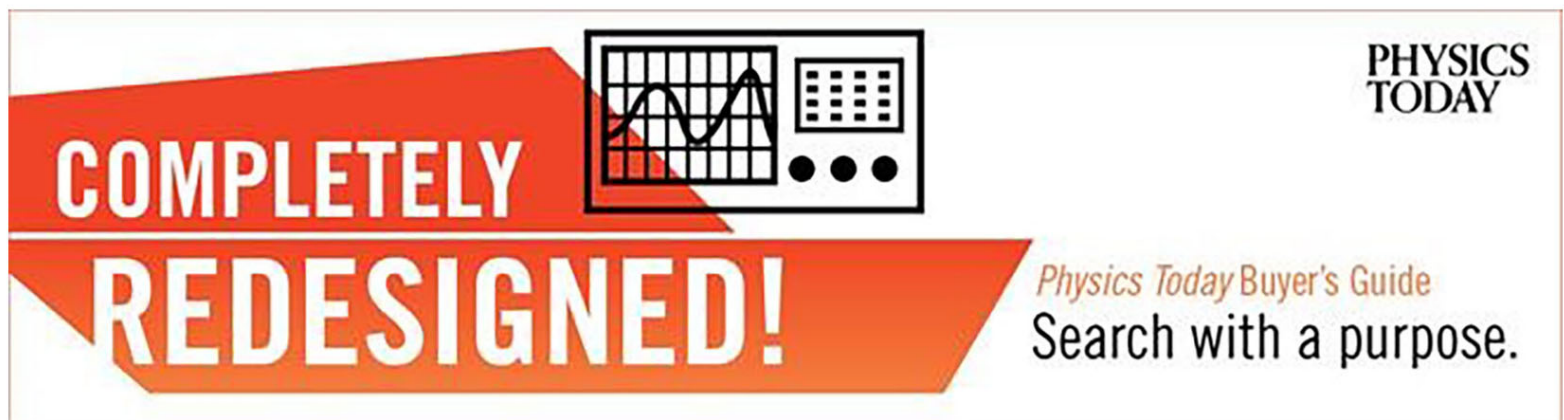




\title{
Saturation process induced by vortex-merging in numerical Vlasov-Maxwell experiments of stimulated Raman backscattering
}

\author{
M. Albrecht-Marc ${ }^{\text {a) }}$ and A. Ghizzo \\ L.P.M.I.A. UMR 7040, Université Henri Poincaré, BP 23954506 Vandoeuvre les Nancy, Cedex France \\ T. W. Johnston \\ I.N.R.S. Energie et Matériaux, CP 1020, Varennes, J3X1S2, Québec, Canada \\ T. Réveillé, D. Del Sarto, and P. Bertrand \\ L.P.M.I.A. UMR 7040, Université Henri Poincaré, BP 23954506 Vandoeuvre les Nancy, Cedex France
}

(Received 8 February 2007; accepted 24 April 2007; published online 25 July 2007)

\begin{abstract}
The influence of low-frequency nonlinear Bernstein-Greene-Kruskal (BGK)-type waves induced by trapped electrons in backward stimulated Raman scattering is investigated in optical mixing. Semi-Lagrangian Vlasov-Maxwell simulations show two nonlinear behaviors. First, there is a Morales-O'Neil plasma wave frequency downshift retuned by a small wavenumber shift which maintains the Stimulated Raman Scattering (SRS) resonance. The saturation of Raman backscattering begins with phase space vortex merging followed by a transition to lower wavenumbers following the (nonlinear) dispersion relation, resembling weak turbulence.
\end{abstract}

(C) 2007 American Institute of Physics. [DOI: 10.1063/1.2749715]

\section{INTRODUCTION}

In this paper we consider the Stimulated Raman Scattering in the Backward direction (SRS-B), sometimes called BSRS, and laser-plasma instabilities involving Electron Plasma Waves (EPWs) in large plasmas in kinetic regimes $\left(k_{B} \lambda_{\mathrm{De}} \geqslant 0.3\right.$, where $k_{B}$ is the Langmuir wavevector for SRS-B and $\lambda_{\text {De }}$ is the electron Debye length), for which an essential role is played by well-trapped electrons. The regimes studied are relevant to the plasma conditions expected in ignition designs to be fielded at the National Ignition Facility (NIF) (see Ref. 1 for more details), as well the Laser MégaJoule project (LMJ) in France (see also Ref. 2, currently under construction).

In the weak Landau regime $\left(k_{B} \lambda_{\mathrm{De}} \leqslant 0.3\right)$, it is well known that the main mechanism responsible for the saturation of SRS-B is the Langmuir Decay instability (LDI), but the evidence that LDI limits the amplitude of SRS-B was only gathered in that regime. In the regime where $k_{B} \lambda_{\mathrm{De}}$ $\geqslant 0.3$ (i.e., at high plasma temperature or low density), referred to here as the kinetic regime of the instability, the situation proves to be more complex. SRS-B reflectivity presents a bursting behavior, building up and breaking down in cycles. Different explanations for this regime were proposed. $\mathrm{Vu}$ et al. in Ref. 3 propose a saturation of SRS by detuning due to the nonlinear frequency shift of the EPW, while Brunner and Valeo in Ref. 4 have suggested the breakup of EPW through the trapped-particle instability. In both models trapped electrons play a major role, so the nonlinear behavior of the plasma is dominated by kinetic effects and not by fluid effects, through an initial fluid phase and its transition to the kinetic one was recently identified by Kline et al. and presented in Ref. 5. At low $k_{B} \lambda_{\text {De }}$ values, LDI was observed via the existence of multiple EPWs while, as $k_{B} \lambda_{\mathrm{De}}$ increases, the

\footnotetext{
${ }^{a)}$ Present address: CEA Bruyeres le Chatel BP12 91680 France.
}

amplitudes of the daughter waves from the LDI cascade drop below the detection threshold and a single frequencybroadened EPW spectrum was observed. It is clear that in that kinetic regime, trapping effects and the associated nonlinear shift in frequency (due to the nonlinear mode coupling or particle trapping) are expected to dominate since the LDI process is too heavily Landau damped to compete. Kinetic effects may however take on different aspects.

A clear indication of kinetic behavior was the recent observations in Refs. 5 and 6 at the Trident laser facility of (SRS-B), associated with what was termed Stimulated Electron Acoustic Scattering (SEAS), a novel scattering apparently involving a so-called Electron Acoustic Wave (EAW), whose phase velocity $v_{\phi} \simeq 1.3 v_{\text {th }}$ is between an EPW and an Ion Acoustic Wave (IAW and $v_{\text {th }}$ being the electron thermal velocity) and whose frequency is a modest fraction of the plasma frequency such as one third. Such waves are justified as the small-amplitude limit of waves which depend essentially on trapped electrons as theoretically predicted by Krapchev and Ram in Refs. 7 and 8 and by Holloway and Dorning in Ref. 9 and are thus quite distinct from usual IAWs, which are linear waves. The concept of these waves are a novel version of a Bernstein-Greene-Kruskal (BGK) (see, for instance, Ref. 10) nonlinear wave with electrons trapped in the wave troughs. This brings us to an important question of the physical origin of these low-frequency kinetic waves. Recently electrostatic Vlasov simulations, using an external independent ponderomotive driver of finite duration have shown that long-lived excitations at frequencies at something like a third of the plasma frequency can indeed be produced (if coherently driven up to self-sustaining level) over a wide range of frequencies for a given wavenumber and have been termed KEEN waves for Kinetic Electrostatic Electron Nonlinear waves in Ref. 11. (Unlike the BGK formulation, however, long-lived fluctuations are seen in the 
co-moving frame.) In another development on this kind of wave, Particle-in-Cell (PIC) simulation studies by Nikolic et al. in Ref. 12 on plasma slabs of modest size have been re-examined by Ghizzo et al. in Ref. 13, but this work is limited to relatively thin plasma slabs rather strongly driven. Even more recent work by Strozzi et al. presented in Ref. 14 points to a likely origin of the SEAS seen by Montgomery et al. (see, for example, Ref. 6 for more details) as a SRS-B by-product involving the EPW spectrum as modified by the space-averaged trapped electron velocity distribution.

The work by Strozzi et al. just cited is the most recent of a series from that of Kline et al. in Ref. 5, including since then Vu et al. in Ref. 15 and Yin et al. in Refs. 16 and 17. This work can be best understood by distinguishing it from the pioneering work of people like Forslund, Kindel, and co-workers in Ref. 18 and of Kruer, Estabrook, and Langdon and co-workers presented in Ref. 19. Those early 1.5D SRS simulations in underdense plasmas were, because of computer limitations, necessarily modest in size (i.e., thickness), and time, and often the intensities were relatively high (to have significant instability with the overall time limitations). The fast forward-going (with respect to the incident laser light) electrons produced by the SRS-induced strong plasma waves would travel to the far boundary (i.e., furthest away from the laser source) and there reflux from (i.e. be returned by) the plasma sheath field from the far-side plasma sheath. These refluxed energetic electrons would then travel back to the near side, reflux again and enter the interaction region to absorb yet more energy from SRS instability and thus tend towards a self-limiting of the SRS activity by the generation of fast electrons. In the larger-scale simulations which can now be done at lower intensities and run for longer times to be closer to Inertial Confinement Fusion (ICF) operating conditions, a somewhat different picture emerges. Long before the electrons accelerated by the SRS instability can return to the SRS growth region going in the same direction as the plasma wave, two other things have happened. First, as discussed by others as given in Refs. 15-17 the plasma wave undergoes a frequency decrease, according to the basic idea of Morales and O'Neil regarding the effect of trapped electrons on the frequency of a Langmuir wave, while the wavevector increases slightly so as to maintain the SRS resonance (see, for example, Refs. 15-17). It had originally been conjectured by Vu et al. in Ref. 3 that the frequency change would act to detune the interaction and at least substantially limit the effects of the instability, but in fact the simultaneous wavevector retuning allows the instability to continue. What then appears to happen is that, instead of a decrease in instability rate due to increasing energy losses as in early, smaller simulations, the SRS-driven retuned EPW interaction continues easily with well-trapped electrons, until arrested by a new process. That new process is the principal object of the present work.

This SRS-limiting process begins with a symmetrybreaking instability of pairwise merging of phase-space "holes" or vortices, an instability which was first seen in simulation of strong two-beam instability by Bertrand et al. in Ref. 22. In our much larger nonuniform plasma system the vortex-merging occurs at similar times and more less ran- domly in space. This vortex-merging symmetry-breaking behavior, which before had been seen only in an ideal uniform electrostatic periodic system, proves to be pertinent even though we are faced here in our Vlasov simulations with a large plasma and with the persistence these phase-space vortices over some thousands of inverse pump frequencies.

This vortex-merging process then evolves towards a resonant spectrum resembling that of a weak electrostatic inverse cascade (i.e., towards lower wavenumbers). This gives a rather chaotic state with a modest range of frequency and a larger range of wavevectors (the observed $k c / \omega_{0}$ values range from the original resonant value of about 1.5 down to 1.0). It is this lack of coherence is what first breaks the resonant SRS coupling.

As implied above we address in this paper the physics that ultimately limits this scattering. We have investigated these phenomena in simulations on two types of underdense plasma, (i) a slab plasma (relatively sharp sides and a flat top) to isolate the physics and (ii) a parabola-shaped plasma to verify that these phenomena are not limited to very uniform plasmas. In general the phenomena are about the same in the two cases. Since we want to show clearly the phasespace vortex structures, rather than using a PIC code, these computer experiments are carried out with a semiLagrangian Vlasov code (first presented in Ref. 21). These simulations have proven to display a very low level of noise compared to PIC codes, which proves particularly advantageous at phase velocities where the space-averaged particle density is low.

\section{BASIC EQUATIONS}

To describe the behavior of an electromagnetic wave propagating in a collisionless plasma, we need to solve the Vlasov equation for each particle species $(\alpha=e, i)$. To model this, we consider an inhomogeneous (parabolic) profile (the case of an homogeneous plasma is also considered) where $n_{0}$ is the value of the electron density on the top of the density profile. Using the Coulomb gauge $\operatorname{div} \mathbf{A}=0$, we restrict the potential vector $\mathbf{A}$ to be in the $y$-direction only (which corresponds to the case of linear polarization). Recalling that

$$
\mathbf{E}=-\nabla \phi-\frac{\partial \mathbf{A}}{\partial t}
$$

we have in the perpendicular direction $y$ :

$$
E_{y}=-\frac{\partial A_{y}}{\partial t}
$$

and in the longitudinal direction

$$
E_{x}=-\frac{\partial \phi}{\partial x} .
$$

We use a bounded (nonperiodic) model which is therefore causal. The distribution function $f_{\alpha}$ for each particle species satisfies the one-dimensional (1D) relativistic Vlasov equation, 


$$
\frac{\partial f_{\alpha}}{\partial t}+\frac{p_{x \alpha}}{m_{\alpha} \gamma_{\alpha}} \frac{\partial f_{\alpha}}{\partial x}+q_{\alpha}\left(E_{x}+\frac{P_{y}(x, t) B_{z}}{m_{\alpha} \gamma_{\alpha}}\right) \frac{\partial f_{\alpha}}{\partial p_{x \alpha}}=0,
$$

where $q_{\alpha}$ is the charge of the particle species $\alpha$.

The Lorentz factor $\gamma_{\alpha}$ is then approximated by

$$
\gamma_{\alpha} \simeq \sqrt{1+\frac{p_{x \alpha}^{2}}{m_{\alpha}^{2} c^{2}}} .
$$

The transverse momentum $p_{y \alpha}=P_{y \alpha}(x, t)$ is simply obtained through the conservation of the generalized canonical momentum

$$
\mathbf{P}_{c}=\mathbf{p}_{\alpha}+q_{\alpha} \mathbf{A}=0
$$

connected to the particle momentum $\mathbf{p}_{\alpha}$ which can be taken to zero without loss of generality.

\section{HOMOGENEOUS SLAB DENSITY PROFILE}

We start here with a homogeneous plasma slab in order to determine the main features of the instability, the homogeneous case allowing a more simple approach in comparison with the case of a parabolic profile. We have normalized the frequency to the pump frequency $\omega_{0}$, the time to the inverse pump frequency $\omega_{0}^{-1}$, and the space variable to $c / \omega_{0}$. Let us now define the simulation set up. Use will be made of the propagating field combination $E^{ \pm}=E_{y} \pm c B_{z}$ to account for wave propagation in different directions (this isolates exactly in the vacuum and approximately in low-density plasma). Nonrelativistic SRS scattered frequency shift is necessarily less than half the incident frequency, and the scattered frequency was thus to be between the incident frequency and half the incident frequency. Since we were to use a triggering source for this signal, the frequency choice was ours to make and was taken to be exactly $2 / 3$ in order to make frequency shifts easy to see. For a cold plasma this would imply a density value of $n_{0}=(1 / 3)^{2} n_{c} \simeq 0.111 n_{c}$.

The plasma slab thickness is $L_{\text {slab }} \omega_{0} / c=3900$ or equivalently $L_{\text {slab }} \simeq 620 \lambda_{0 \mathrm{vac}}$, which is about $220 \mu \mathrm{m}$ (if we take the pump wavelength $\lambda_{0 \mathrm{vac}}$ in vacuum to be the usual third harmonic of a neodymium laser, i.e., about $0.35 \mu \mathrm{m})$. The plasma will be initially assumed to be Maxwellian with an electron temperature of $T_{e}$ of $2 \mathrm{keV}$. This nonzero temperature means that perfect phase matching now implies a density of $n_{0} \simeq 0.0825 n_{c}$, rather than the cold plasma value of $n_{0} \simeq 0.111 n_{c}$. With the plasma density and temperature thus defined the plasma slab width in Debye lengths is $L_{\text {slab }} \simeq 18000 \lambda_{D}$.

We begin with a simulation of only SRS-B with injected counterpropagating pump $\left(\omega_{0}, k_{0}\right)$ and a seed $\left(\omega_{s B}, k_{s B}\right)$ of $(2 / 3) \omega_{0}$ probe light with intensities of $I_{\text {probe }}=10^{-4} I_{\text {pump. }}$. The total length of the plasma is indeed $L=L_{\text {slab }}+2 L_{\mathrm{vac}}+2 L_{\text {jump }}$; $L_{\text {slab }}$ is the length over which the plasma is uniform, $L_{\mathrm{vac}}$ is the length of the vacuum on both sides of the plasma and $L_{\text {jump }}$ is a small length over which the density jumps from 0 to $n_{0}$. Here we have used $L_{\mathrm{vac}}=48 \mathrm{c} / \omega_{0}$ and $L_{\text {jump }}=50 \mathrm{c} / \omega_{0}$. The total length of the computational plasma box is then $L=4096 \mathrm{c} / \omega_{0}$ corresponding to $624.4 \lambda_{0 \mathrm{vac}}$ or about $595 \lambda_{0}$, where $\lambda_{0}$ is the pump wavelength in the plasma (the refractive index being 0.96 for $n / n_{c}=0.0825$ ). We have used a
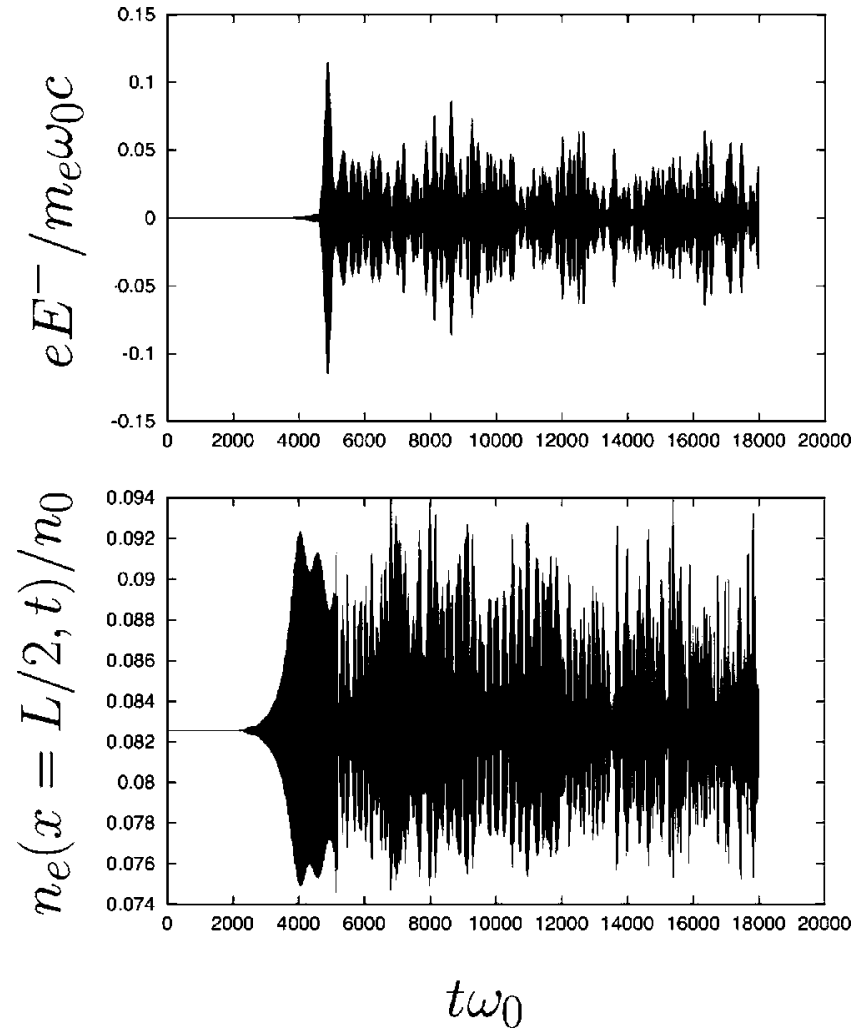

FIG. 1. For the uniform slab plasma (maximum density of $n_{0} / n_{c}=0.0825$, $a_{0}=0.025$ and $T_{e}=2 \mathrm{keV}$ ), the typical temporal behavior is shown for (a) the scattered mode $e E^{-} / m_{e} \omega_{0} c$ (top panel), as recorded in the vacuum at the left plasma boundary, and (b) for the density evolution in the middle of the plasma (bottom panel). Two different phases are evident: a fluid (coherent) phase at the beginning of the evolution (for $t \omega_{0} \leqslant 5000$ in the plasma, and $t \omega_{0} \leqslant 5600$ for the backscattered signal at the left boundary) followed by the "burst-like" regime for later times.

sampling of $N_{x} \times N_{p_{x}}$ of $16384 \times 256$ grid points in phase space which corresponds to a grid spacing of $\Delta x \omega_{0} / c=0.25$ and mesh size of $\Delta p_{x}=7.8 \times 10^{-3} m_{e} c$ in momentum.

The quiver momentum of the pump wave is $a_{0}=p_{\text {osc }} / m_{e} c=0.025$ which corresponds to an intensity of $I_{\text {pump }} \simeq 7 \times 10^{15} \mathrm{~W} \mathrm{~cm}^{-2}$. Ions are immobile in this simulation.

We recall the frequency and wavenumber matching conditions between waves in the the plasma for SRS-B instability as $\omega_{0}=\omega_{s B}+\omega_{B}$ and $k_{0}=-k_{s B}+k_{B}$. Normalizing frequencies to the pump frequency $\omega_{0}$, we have $\omega_{s B}=2 / 3 \omega_{0}$, $\omega_{B}=1 / 3 \omega_{0}$, and $k_{0} c / \omega_{0}=0.9578$ (for the pump wave), $k_{s B} c / \omega_{0}=0.6016$ (for the seed) and $k_{B} c / \omega_{0}=1.5594$ for the EPW, which corresponds to a value of $k_{B} \lambda_{\mathrm{De}} \simeq 0.34$, well into the kinetic regime.

Let us first examine the temporal behavior of the amplified probe signal (the scattered mode) and the corresponding plasma mode is shown in Fig. 1. We clearly distinguish between two different phases. The first one $\left(t \omega_{0} \leqslant 5000\right)$ corresponds to the fluid phase. This phase shows rather coherent shapes of both modes and corresponds to weak kinetic effects. After this phase ends, we recover the expected bursting behavior of SRS-B, already predicted in Ref. 3 and in Ref. 4 in both panels. To characterize this behavior in the plasma, namely what these kinetic effects are, it is useful to look at 

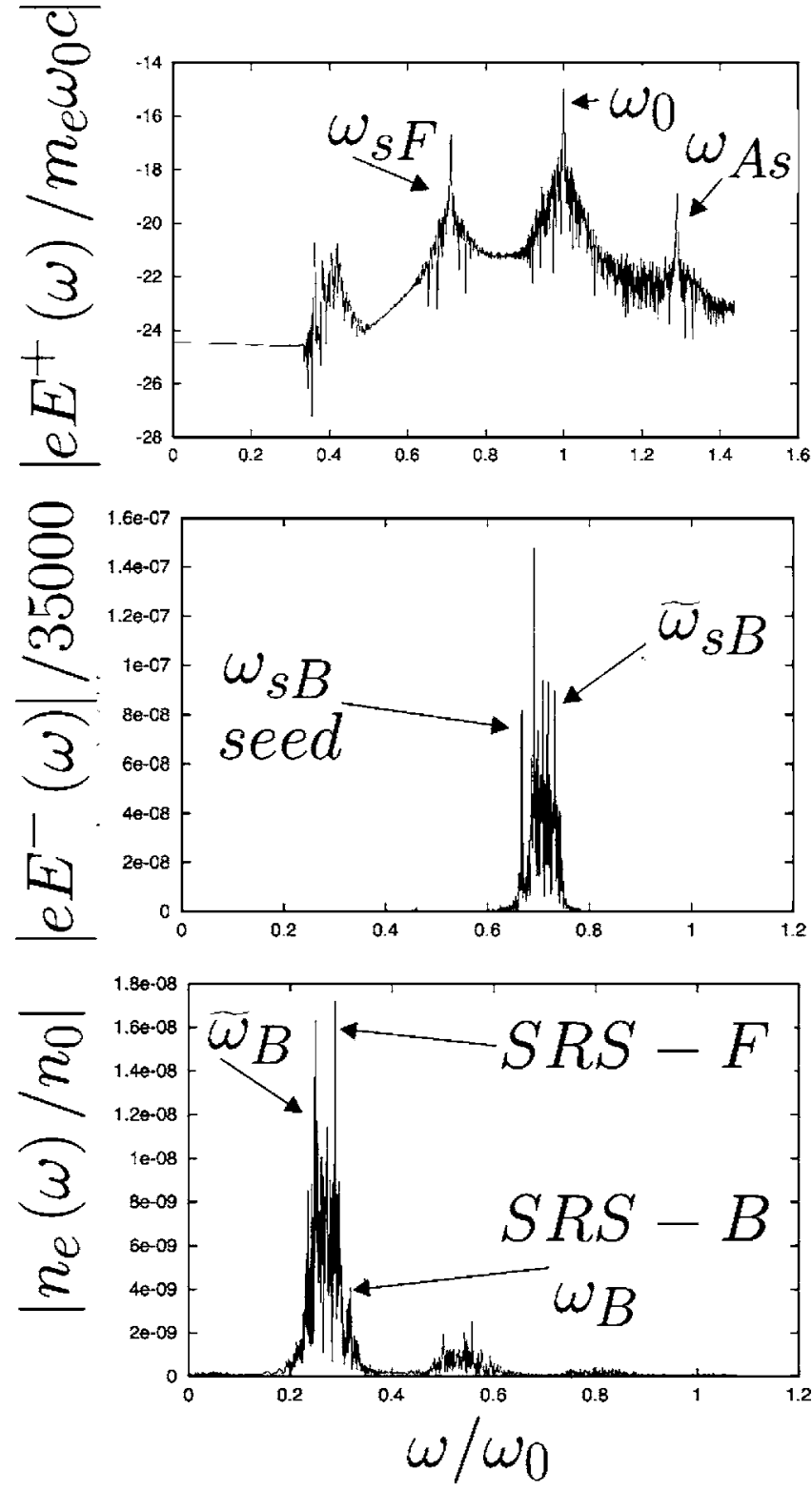

FIG. 2. For the uniform slab plasma the frequency spectra are shown of (a) the transmitted light (logarithmic scale, top panel), (b) the backscattered Raman light (linear scale, middle panel), and finally (c) the electron density $n_{e} / n_{c}$ (linear scale bottom panel). The time over which the spectra were taken was for $t \omega_{0}$ values between (7000 and 16000).

the spectra of these modes in a time interval located in the bursting phase, i.e., from $t \omega_{0}=7000$ to $t \omega_{0}=16000$.

In Fig. 2 we have plotted the $\omega$-spectrum amplitude of the forward field $e E^{+} / m_{e} \omega_{0} c$ (top panel), the backscattered contribution of the light, i.e., $e E^{-} / m_{e} \omega_{0} c$ (middle panel) and of electron density $n_{e}$ at the middle of the system (bottom panel). The nonlinear shift in frequency induced by trapping effects was observed to have its upper limit at $\widetilde{\omega}_{s B}=0.75 \omega_{0}$ (rather than the original seed value of 0.666 ) for the scattered light and the corresponding downshifted limit value from the $n_{e}$ spectra to be $\widetilde{\omega}_{B}=0.25 \omega_{0}$ for EPW rather than the original value of 0.333 . We denote here by $(\widetilde{\omega}, \widetilde{k})$ the new values of the frequency and wavenumber of the shifted wave.

While the frequency shift was indeed seen in Ref. 3, the saturation of instability due to the detuning as predicted in
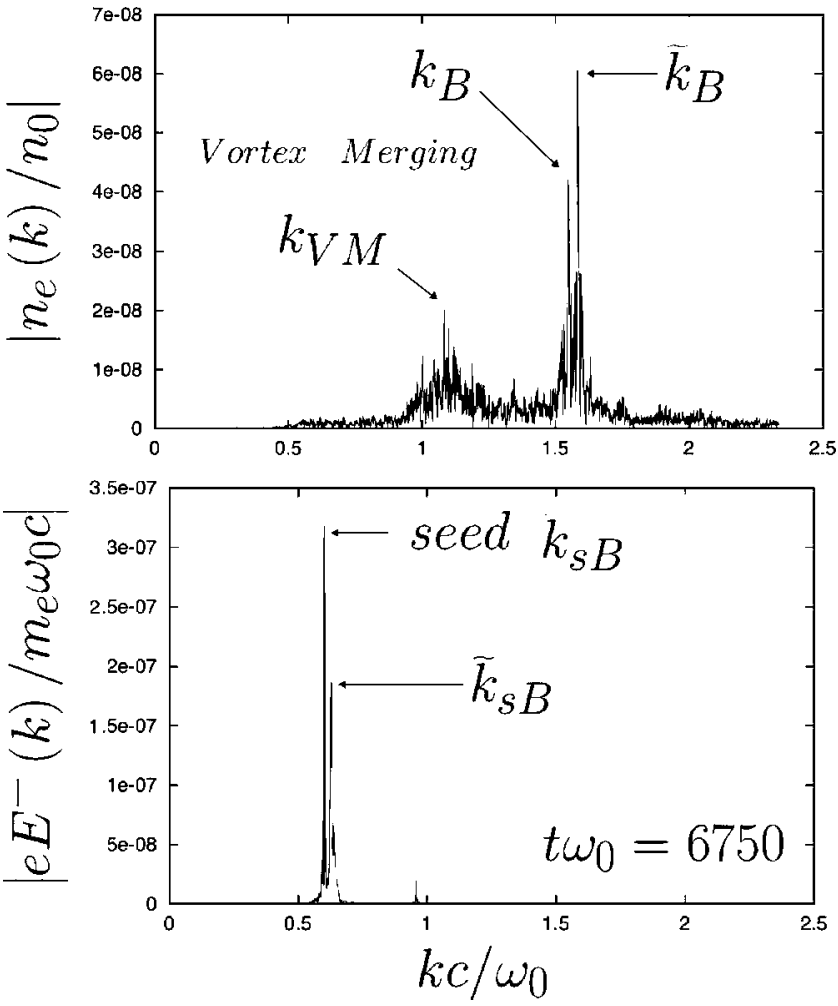

FIG. 3. For the uniform slab plasma of Figs. 1 and 2, at time $t \omega_{0}=6750$ (i.e., after the burst-like phase has begun), the $k$-spectra of the electron density (in top panel) and the backscattered electromagnetic field (in bottom panel). Notice the increase in the wavenumbers for both spectra from the initial $k_{B}$ and seed $k_{s B}$ values to the larger final values of $k_{\mathrm{BLMO}}$ and $k_{s \mathrm{BLMO}}$, respectively. This is due (see text) to a Langmuir Morales-O'Neil (LMO) shift with intensity.

Ref. 3 was not observed in optical mixing. This is because there is a corresponding shift for wavevectors (as can be seen for instance in Fig. 3) which means that matching conditions are maintained in spite of the shift and become here $\omega_{0}$ $=\widetilde{\omega}_{s B}+\widetilde{\omega}_{B}$ (here $1=0.75+0.25$ in $\omega_{0}$ units) and for wave numbers $k_{0}=-\widetilde{k}_{s B}+\widetilde{k}_{B}$ (here $0.9578=-0.6078+1.65$ in $\omega_{0} / c$ units). The pump wave frequency and wavevector of course remain unmodified as a constant source of energy. Therefore, due to a constant retuning of the phase matching relations, this new set of matching relations allows the instability to persist in time more than it should if the frequency shift at a fixed wavenumber was a source of instability saturation.

The shift in frequency and wavevector is chiefly a nonlinear phenomena and it is important to understand its effect on the EPW. To summarize what happens in various regions of the plasma, let us consider the data of Figs. 3 showing, at a given time the $k$-spectra of the electron density (in top panel) and the backscattered electromagnetic (in bottom panel).

Figure 3 is given at the time $t \omega_{0}=6750$. This is the end of the fluid-like phase. The main plasma is now subject to the bursting behavior. However at the right side of the system, the plasma is still unperturbed. This region in fact exhibits very regular trapping structures and contributes to the value of $k_{B} c / \omega_{0} \simeq 1.559$ in the spectrum. Figure 3 gives an example where both wavevectors $\left(k_{B}\right.$, the initial value, and $\widetilde{k}_{B}$, 
the upshifted value) are present, at the same time in plasma (but not located at the same position); the shifted value $\widetilde{k}_{B}$ thus corresponds to the "turbulent" region of the plasma.

Unlike the original initial-value problem of Morales and O'Neil (see, for instance, Ref. 20) where the wavevector was fixed at the initially imposed value, here both the frequency and wavevector change (or "chirp") downward in frequency for EPW (giving $\widetilde{\omega}_{B}$ ) and upward in the wavevector. In this way, the scattered electromagnetic mode keeps on satisfying its linear dispersion relation while the electrostatic plasma mode does not, so much so that its frequency actually drops below the plasma frequency, i.e., $\widetilde{\omega}_{B}=0.25 \omega_{0} \leqslant \omega_{p e}$ $\simeq 0.287 \omega_{0}$. As a consequence, there is a change in the nature of this initially EPW, which is no longer a simple Langmuir wave at later times, but, as we hypothesize, a more complex structure characterized by trapping effects in the form of phase space holes. These new waves were first generated as classic Langmuir waves associated with SRS-B and propagating away from the laser, but with normalized frequency and wavevector here nonlinearly lowered by generalized Morales-O'Neil retuning become a structure we characterize here as Generalized-Morales-O'Neil (GMO), here with normalized frequency and wavevector values of $\widetilde{\omega}_{B} / \omega_{0}=0.25$ and $\tilde{k}_{B} c / \omega_{0} \simeq 1.66$, compared with the initial values of $\widetilde{\omega}_{B} / \omega_{0}=0.333$ and $\widetilde{k}_{B} c / \omega_{0} \simeq 1.559$. (We emphasize that although initial-value states such as those considered in the original analysis are hard to create experimentally, the basic Morales-O'Neil concept is nonetheless at the heart of what is seen in the SRS-B instability in a totally different context.)

These phase-space vortex structures eventually turn out to be unstable and not obviously self-sustained and are apparently able to merge together in a pairwise vortex merging process. Some evidence for that process can be seen in Fig. 3 (top panel) on the electronic density $k$-spectrum. Not only can we clearly see the nonlinear shift, but we also observe the presence of a rather broad (and at lower levels) spectrum located around $k_{\mathrm{VM}^{c}} c / \omega_{0} \sim 1$ that is the consequence of the vortex merging phenomena in the plasma. It seems as if the trapping structures (or phase space holes) generated by previously strong SRS-B are now experiencing a weaker driver and thus start to merge as a natural mechanism. We will discuss that phenomenon further later on in Sec. VI.

\section{FREQUENCY VERSUS WAVENUMBER BEHAVIOR OF SRS-B}

Since the spectrum is a result of the interplay of the time-dependent trapping shift of EPW and maintains the parametric resonance (here SRS-B), let us now consider for the same simulation a different diagnostic approach based on spectrum analysis in the $k$ - $\omega$ plane, the well-known Brillouin diagram.

The wavevector behavior of the appropriate quantity was obtained by first taking a spatial Fast Fourier Transform over the plasma slab length of $L_{\text {slab }} \omega_{0} / c=3900$ at each time step in the range from zero to $t \omega_{0} / c=21000$, and then taking the time Fast Fourier Transform (FFT) for each wavenumber component. The magnitude of the results are displayed in the Brillouin diagram format of $\omega / \omega_{0} \mathrm{vs} k c / \omega_{0}$. The backscat-
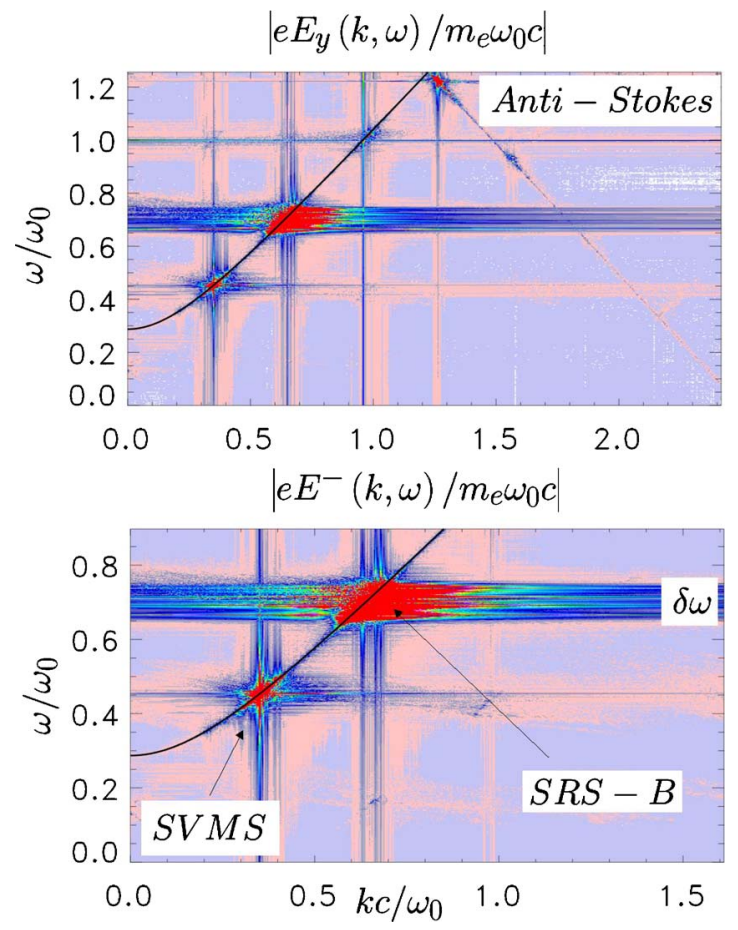

FIG. 4. (Color online) For the uniform slab plasma simulation of Figs. 1 and 2, we show here the total $\left(E_{y}\right)$ electromagnetic spectrum in the top panel and a zoom of the backscattered contribution $\left[\left|e E^{-}(k, \omega) / m_{e} \omega_{0} c\right|\right]$ in the bottom panel. The solid line curve corresponds to the linear dispersion relation of the electromagnetic wave in plasma. The spectral features of SRS-B (i.e., $\omega$ and $k$, which shift together to stay on the electromagnetic plasma dispersion relation) are the combined result of the generalized LMO nonlinear resonance shift due to the trapping and the maintenance of the parametric resonance lock in the optical mixing. The SVMS feature at the lower $(\omega, k)$ combination corresponds to a rescattering from SRS-F via the vortexmerged contribution to the electrostatic spectrum. However pump scattering from the second harmonic of the nonlinear electrostatic plasma wave is also possible (as shown in Fig. 6).

tered electromagnetic wave spectrum $\left|e E^{-}(k, \omega) / m_{e} \omega_{0} c\right|$ is plotted in Fig. 4 in the bottom panel. In the top panel we have also shown the spectrum of the $y$-component of the electromagnetic field. The electromagnetic spectrum of Fig. 4 are the combined result of the nonlinear shift due to the trapping and the parametric resonance which is rigorously maintained in time in the optical mixing scenario.

First, as the frequencies shift for the plasma wave $(B)$ and the scattered wave $(s)$, we get also a shift for the wavevectors such that the three-wave $[\operatorname{wave}(0)=$ wave $(s)$ +wave $(B)$ ] matching relations in both frequencies and wavevectors are satisfied over time.

Starting from the matching condition in frequency, we obtain

$$
\omega_{0}=\widetilde{\omega}_{s B}+\widetilde{\omega}_{B}=\omega_{s}\left(k_{0}-k_{B}-\delta k\right)+\omega_{B}-\delta \omega
$$

we have $k_{0}=-\widetilde{k}_{s B}+\widetilde{k}_{B}=-\widetilde{k}_{s B}+k_{B}+\delta k$ and we have assumed that $\widetilde{k}_{B}=k_{B}+\delta k$ and $\widetilde{\omega}_{B}=\omega_{B}-\delta \omega$. Here $\omega_{s B}(k)$ is the scattered wave frequency written as a function of its wavevector $k$. Here we have $-k_{s B}=k_{0}-k_{B}$ (i.e., $-0.6016=0.9578-1.5594$ ). The linear matching condition in frequencies is then given by $\omega_{0}=\omega_{s B}+\omega_{B}$. The variation in frequency is close to $\delta \omega=\widetilde{\omega}_{s B}-\omega_{s B}=0.75 \omega_{0}-0.666 \omega_{0} \simeq 0.083 \omega_{0}$. This variation is 


\section{$t \omega_{0}=9000$}
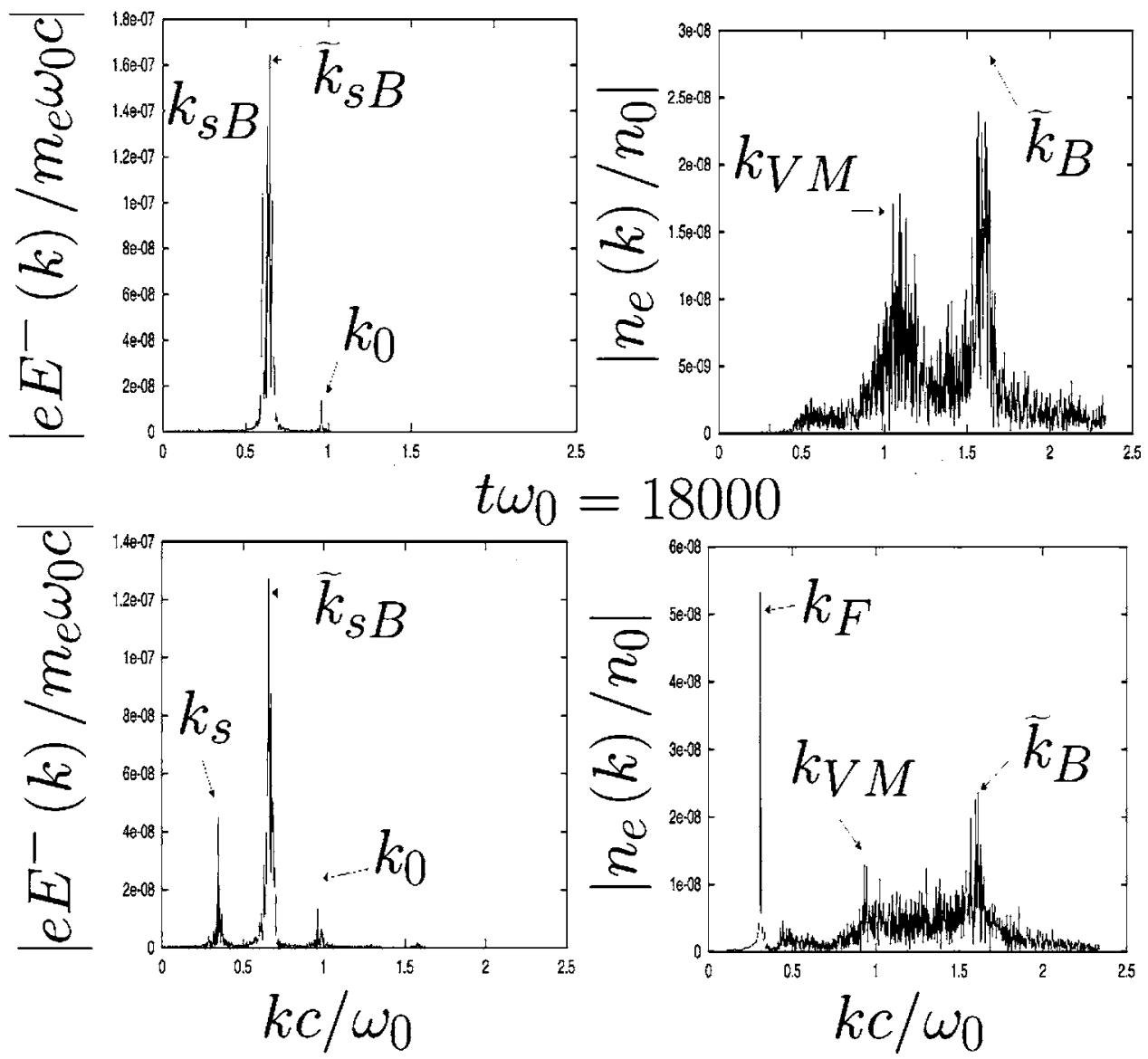

FIG. 5. For the uniform slab plasma, $k$-spectra of the electron density $\left|n_{e}(k, t) / n_{0}\right|$ and of the electromagnetic backscattered contribution $\left|e E^{-}(k, t) / m_{e} \omega_{0} c\right|$ as a function of $k c / \omega_{0}$, and at two different instants during the plasma evolution. At time $t \omega_{0}=9000$, the signature of this rescattering is not visible although the electron density spectrum broadens over a wide domain. The electromagnetic rescattered (backscattered) emission starts only when the SRS-F process begins to occur. The bottom panels show what happens when SRS-F is now well established in the plasma: we observe clearly the growth of the rescattered signal by SRS-F at time $t \omega_{0}=18000$. clearly visible in both spectra in Fig. 4. For small values of $\delta k$ we can write

$$
\widetilde{\omega}_{s B}=\omega_{s B}\left(-k_{s B}-\delta k\right) \simeq \omega_{s B}\left(-k_{s B}\right)-\left.\delta k \frac{\partial \omega_{s B}}{\partial k}\right|_{k=k_{s B}}
$$

or equivalently

$$
\tilde{\omega}_{s B}=\omega_{s B}-v_{g s B} \delta k,
$$

where $v_{g s B}$ is the linear group velocity of the electromagnetic scattered (probe) wave close to $v_{g s B}=k_{s B} c^{2} / \omega_{s B} \simeq 0.92 c$. Here $\tilde{\omega}_{s B}$ and $\tilde{k}_{s B}$ satisfy the usual dispersion relation $\widetilde{\omega}_{s B}^{2}=\omega_{p e}^{2}+\widetilde{k}_{s B}^{2} c^{2}$ where $\omega_{p e}=\sqrt{n_{0} e^{2} / m_{e} \epsilon_{0}}$ is the electron plasma frequency. The group velocity may be estimated to

$$
v_{g s B}=\frac{\omega_{B}-\widetilde{\omega}_{B}}{k_{B}-\tilde{k}_{B}}=\frac{\widetilde{\omega}_{s B}-\omega_{s B}}{\tilde{k}_{s B}-k_{s B}}=\left|\frac{\partial \omega_{s B}}{\partial k_{s B}}\right|=0.92 c .
$$

In addition to this behavior an unusually interesting feature can also be noticed in Fig. 4 on the plasma electromagnetic dispersion locus at about $\omega / \omega_{0}=0.45$ (note that this also seen in the spectra of the upper panel of Fig. 2) and $k c / \omega_{0}=0.37$. Clearly, since the normalized frequency difference of this wave frequency with the pump frequency is 0.55 , which is far larger than the normalized plasma frequency of 0.287 (the square root of $n_{\max } / n_{c}=0.0825$ ), some more complicated explanation is required. Two candidates suggest themselves.
One explanation involves the Forward Stimulated Raman Scattering (SRS-F) which is seen in the $\left|e E^{+}(\omega) / m_{e} \omega_{0} c\right|$ forward wave electromagnetic frequency spectrum shown in the top panel of Fig. 2, with a frequency of about $\omega_{s F}=0.710 \omega_{0}$, which is also shown with its wavevector of $k_{s F} c / \omega_{0}=0.650$, in the $\left|e E_{y}(k, \omega) / m_{e} \omega_{0} c\right|$ upper panel of Fig. 4 (there overlapped with the backscattered SRS-B). We can write the frequency and wavevector matching for SRS-F in the form $\omega_{0}=\omega_{s F}+\omega_{F}$ and $k_{0}=k_{s F}+k_{F}$. The values of the corresponding EPW associated with SRS-F (for Forward Stimulated Raman Scattering) from the linear dispersion relation are then $\omega_{F}=0.289 \omega_{0}$ for the frequency and $k_{F} c / \omega_{0}=0.308$ for the wavevector, and these are indicated with the SRS-F label shown in the $\left|e E_{x}(k, \omega) / m_{e} \omega_{0} c\right|$ results in Fig. 6). With these data, one hypothesis for the backscatter feature with $\omega / \omega_{0}=0.46$ and $k c / \omega_{0}=0.37$ is rescattering of SRS-F light by the feature associated with the velocity-merging followed by the apparent inverse cascade process. Here the light was created by SRS-F, with the wave characteristics $\left(\omega_{s F}, k_{s F}\right)$, scatters off the kinetic or vortex-merging (here VM) mode with parameters given by $\omega_{\mathrm{VM}}=0.25 \omega_{0}$ and $k_{\mathrm{VM}} c / \omega_{0} \simeq 1$, into a backward-propagating electromagnetic light wave with $\omega_{s}=0.46 \omega_{0}$ and $k_{s} c / \omega_{0}=0.36$, as seen in Fig. 5. Frequency and wavenumber matching are then $\omega_{s F} / \omega_{0}=0.71$ $=\omega_{s} / \omega_{0}+\omega_{\mathrm{VM}} / \omega_{0}=0.46+0.25$ and $k_{s F} c / \omega_{0}=0.65=-k_{s} c / \omega_{0}$ $+k_{\mathrm{VM}} c / \omega_{0}=-0.37+1.0$.

While this is all very plausible, the presence in the upper 


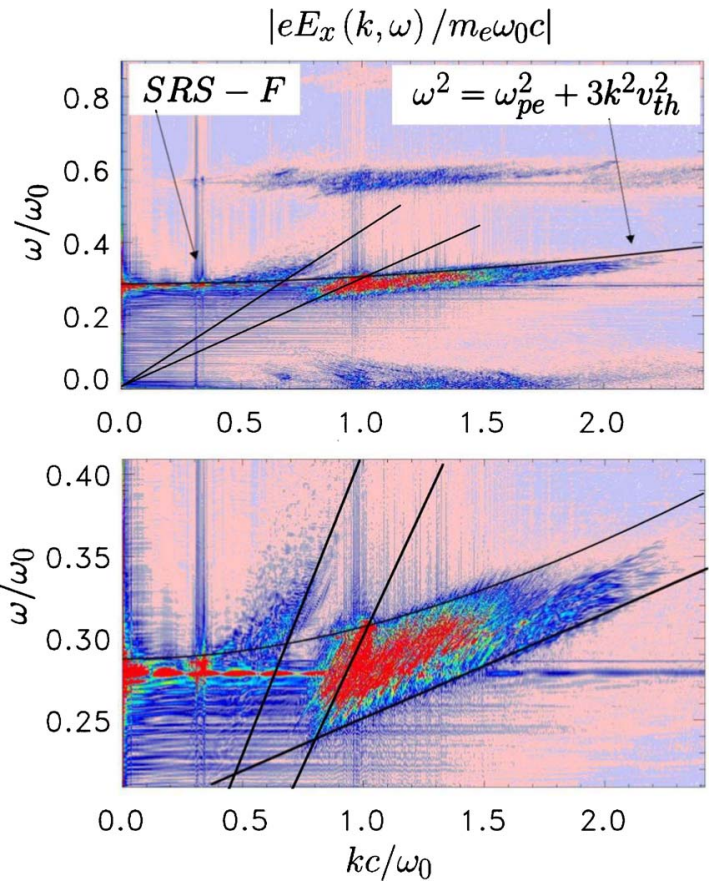

FIG. 6. (Color online) For the uniform slab plasma simulation of Figs. 1 and 2 , we show here the total electrostatic Brillouin diagram ( $\omega$ vs $k$ ) $\left[\left|e E_{x}(k, \omega) / m_{e} \omega_{0} c\right|\right]$ spectrum (top) and a zoom of its structure near the plasma frequency (bottom). Both curves indicate considerable broadening of spectra with frequencies significantly less than the linear dispersion relation as marked by the EPW dispersion curve. The spectra are asymmetrically broadened towards lower Bohm-Gross frequencies, consistent with the trapping-induced LMO frequency shift and lie on the linear curve whose slope is close to the (linear) group velocity of the Langmuir wave as indicated by the lower straight line. Also, modes resembling the "Beam Acoustic Modes" (Ref. 15) and the related beam mode are seen as labelled, and as seen also in other work (Ref. 14). Also, as noted by Strozzi et al. (Ref. 14) this group velocity segment is also close to the phase velocity of what has been termed the EAW or Electron Acoustic Wave (Ref. 6). The source of the initial $\left(\omega_{B}, k_{B}\right)$ scattering is not prominent in these late-time spectra.

(large-range) panel of Fig. 6 in $\left|e E_{x}(k, \omega) / m_{e} \omega_{0}\right|$ of an apparent second frequency harmonic (i.e., $\omega / \omega_{0} \simeq 0.6$ ) of the modified EPW spectrum $\left(\omega / \omega_{0} \simeq 0.3\right)$ means that one should also consider the direct pump scattering from this nonlinear density variation with a wide variation available for $k$, including the $2 \mathrm{xEPW}$ resonance value of (roughly) $\omega / \omega_{0}$ $=0.64$ and $k c / \omega_{0} \simeq 1.37$.

To further investigate this point, we have also represented in Fig. 5 the time-varying corresponding $k$-spectra of the electron density $\left|n_{e}(k, t) / n_{0}\right|$ and of the electromagnetic backscattered contribution $\left|e E^{-}(k, t) / m_{e} \omega_{0} c\right|$ as a function of $k c / \omega_{0}$, and at two different instants during the plasma evolution. As can be seen clearly in the upper two panels of Fig. 5 at the relatively early time $t \omega_{0}=9000$, the signature of this rescattering is not visible although the electron density spectrum broadens over a wide domain. The electromagnetic rescattered (backscattered) emission starts only when the SRS-F process begins to occur. At late time, such as for the lower panels of Fig. 5, one sees what happens when SRS-F is now well established in the plasma; we clearly observe the growth of the rescattered signal from SRS-F. The coupling of this (now existing at a somewhat sufficient level) of the electro-
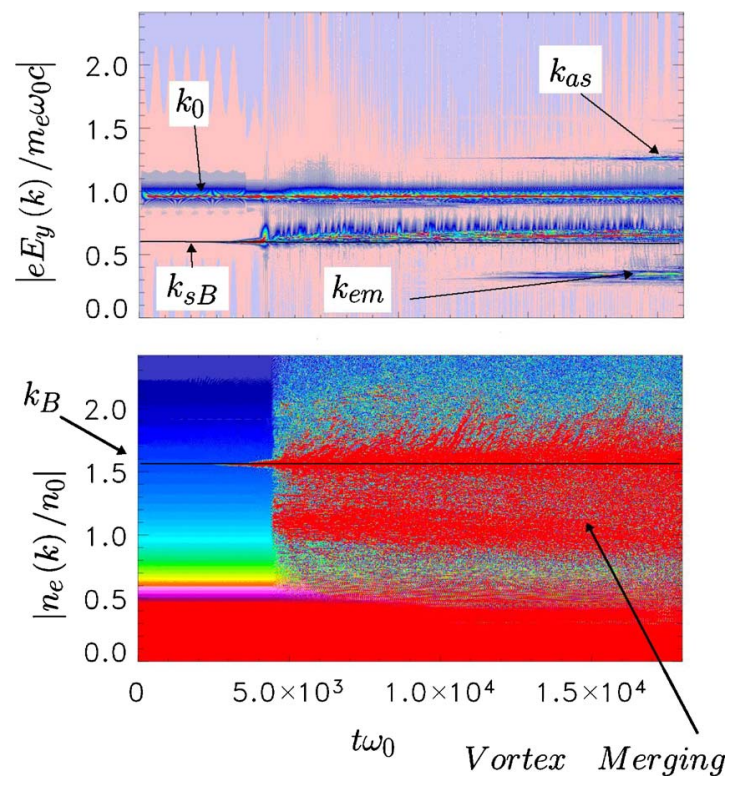

FIG. 7. (Color online) For the uniform plasma slab case the time evolution is shown for the $k$-spectrum of the electromagnetic field (top panel) and for the electron density (bottom panel). The generalized Morales-O'Neil shift in electromagnetic scattering wavevector is clearly visible, the initial value of $k_{s B} c / \omega_{0} \simeq 0.60$ being upshifted to a final value of $\tilde{k}_{s B} c / \omega_{0} \simeq 0.70$. The bottom panel shows the beginning of the turbulent phase (near $t \omega_{0}=5000$ ) characterized by the vortex merging followed by the inverse electrostatic cascade process towards the Beam Acoustic Mode $(k, \omega)$ "knee" near $k c / \omega_{0} \simeq 1.0$ (Refs. 14 and 16).

magnetic backscattered mode with the nonlinear density fluctuations, available in a large range is now possible.

We now turn to other features the longitudinal electric field spectrum $\left|e E_{x}(k, \omega) / m_{e} \omega_{0} c\right|$ of Fig. 6. Both panels show a fairly broad plasma-wave-like spectra but with frequencies significantly less than the linear dispersion relation, not inconsistent with the trapping-induced frequency shift. The spectrum does seem to lie on the linear curve whose slope (estimated numerically to $0.06 c$ ) is close to the expected (linear) group velocity $v_{g B}=3 k_{B} v_{\mathrm{th}}^{2} / \omega_{B}=0.054 \mathrm{c}$. This seems to indicate that, in the inverse-cascade-style process which follows the Langmuir Morales O'Neil (here referred as LMO) shift and the initial vortex-merging events, the waves are not in any sense locked to the original wave. In effect the inverse cascade members satisfy an LMO-shifted dispersion relation similar in form to the original Bohm-Gross relation (equivalent perhaps to a lowered effective plasma frequency) and become incoherent. In addition to these points, forms are seen such as Beam Acoustic Modes and Damped Beam Modes much like those shown by $\mathrm{Vu}$ et al. in Ref. 15, Yin et al. in Refs. 16 and 17, and Strozzi et al. in Ref. 14 to which works the reader is referred to for further information.

In Fig. 7, one finds the time evolution of the electromagnetic field and on the electron density wavenumber spectrum in, respectively, the top panel $\left(\left|e E_{y}(k, t) / m_{e} \omega_{0} c\right|\right)$ and the bottom panel $\left(\left|n_{e}(k, t) / n_{0}\right|\right)$. In the electromagnetic spectrum for the component of the scattered mode, the modest LMO shift in its wavevector is clearly visible, as the initial value of the probe $k_{s B} c / \omega_{0}=0.6016$ is upshifted (between $t \omega_{0}=7500$ to 11000 or so) to $\widetilde{k}_{s B} c / \omega_{0} \simeq 0.70$. In addition one can readily 
note the appearance at $t \omega_{0} \simeq 15000$ of the anti-Stokes mode $\tilde{k}_{\text {as }} c / \omega_{0} \simeq 1.25$. At about the same time the mode with $k c / \omega_{0}=0.37$ appears, associated with $\omega / \omega_{0}=0.46$; this is the low-frequency electromagnetic feature of Fig. 4 whose generation is discussed above. The $\left|n_{e}(k, t) / n_{0}\right|$ in the bottom panel of Fig. 7 shows the beginning of the turbulent phase appearing suddenly somewhat after $t \omega_{0}=5000$. The lower part shows a moderately broad spectrum whose peak decreases from the initial value of $k_{B} c / \omega_{0} \simeq 1.55$ (via what we have termed the apparent inverse cascade) to a final value of $k_{B} c / \omega_{0} \simeq 1.0$. It seems likely that the equivalent LMOshifted plasma wave $(\omega, k)$ spectrum seen in Fig. 6 may well represent a downward chirp rather than the wider spectrum implied in concepts such as "inverse cascade." (Perhaps more sophisticated spectral diagnostics could elucidate this behavior in future work.)

To aid in understanding what is happening, we present in Fig. 8 snapshots of the electron distribution function in phase space $\left(p_{x}\right.$ vs $\left.x\right)$ plots, for various times $\left(t \omega_{0}=4200,8400\right.$, 12600 , and 16800). The $x$-space has been divided in 64 cells with each cell having a length of $64 c / \omega_{0}$, moving at about the phase velocity of the phase space holes. The top panel of Fig. $8\left(t \omega_{0}=4200\right)$ clearly exhibits the trapping and formation of holes (with plasma momentum of 0.218) induced by the EPW in the initial state of the instability with a wavevector close to the expected value of $k_{B} c / \omega_{0} \simeq 1.559$. This cell was selected among all the cells at the same time by considering that the wave train motion is made at the linear phase space velocity. We have also added an arrow on the different images indicating the position of a phase-space marker which would be stationary in the frame moving at the (EPW) phase velocity, i.e., at $v_{\phi}(\mathrm{EPW})=\omega_{B} / k_{B}=0.214 c$.

The phase space vortices allow a convenient immediate perception of the bounce time $\tau_{b}$ from the trapping frequency $\omega_{b}=\left(\Delta p / 2 m_{e}\right) k=\left(\Delta p / 2 m_{e} v_{\text {phase }}\right) \omega_{B}$. This is just $\omega_{b}=\left(\Delta p / 2 m_{e} c\right)\left(c / v_{\text {phase }}\right)\left(\omega_{B} / \omega_{0}\right) \omega_{0}$. Putting in the numbers gives $\omega_{b} \simeq(0.15 / 2)(1 / .2)(1 / 3) \omega_{0} \simeq 0.125 \omega_{0}$, or the trapping frequency is about one-eighth of the pump frequency. Thus the normalized trapping period $\tau_{b} \omega_{0}$ is only $\tau_{b} \omega_{0}$ $=2 \pi\left(\omega_{0} / \omega_{b}\right) \simeq 16 \pi \simeq 50$, which is thus relatively short compared with the evolution time of the system. A great deal of kinetic evolution is thus possible.

Somewhat before the time $t \omega_{0}=8400$ (second panel of Fig. 8), the system has undergone a transition to a more turbulent regime as reflected in the chaotic electron phase space structure resulting from sequential and (apparently) pairwise coalescence in phase space. The third panel in Fig. 8 shows the electron distribution function at rather later time $\left(t \omega_{0}=12600\right)$. The cell is now located around the position $2646 c / \omega_{0}$. We clearly see the maintenance in time of the trapping structures, but now with the wavevector magnitude in the vicinity of $k_{\mathrm{VM}} c / \omega_{0} \sim 1$ rather than the initial value of about 1.6. At later time the situation is more complex since now SRS-F occurs and modulates the electron distribution function on the wavevector $k_{F} c / \omega_{0} \sim 0.3$. As the system evolves, SRS-F becomes significant as shown in the right bottom panel in Fig. 5. Note that at previous time $\left(t \omega_{0}=9000\right)$ SRS-F is not visible in the spectrum.

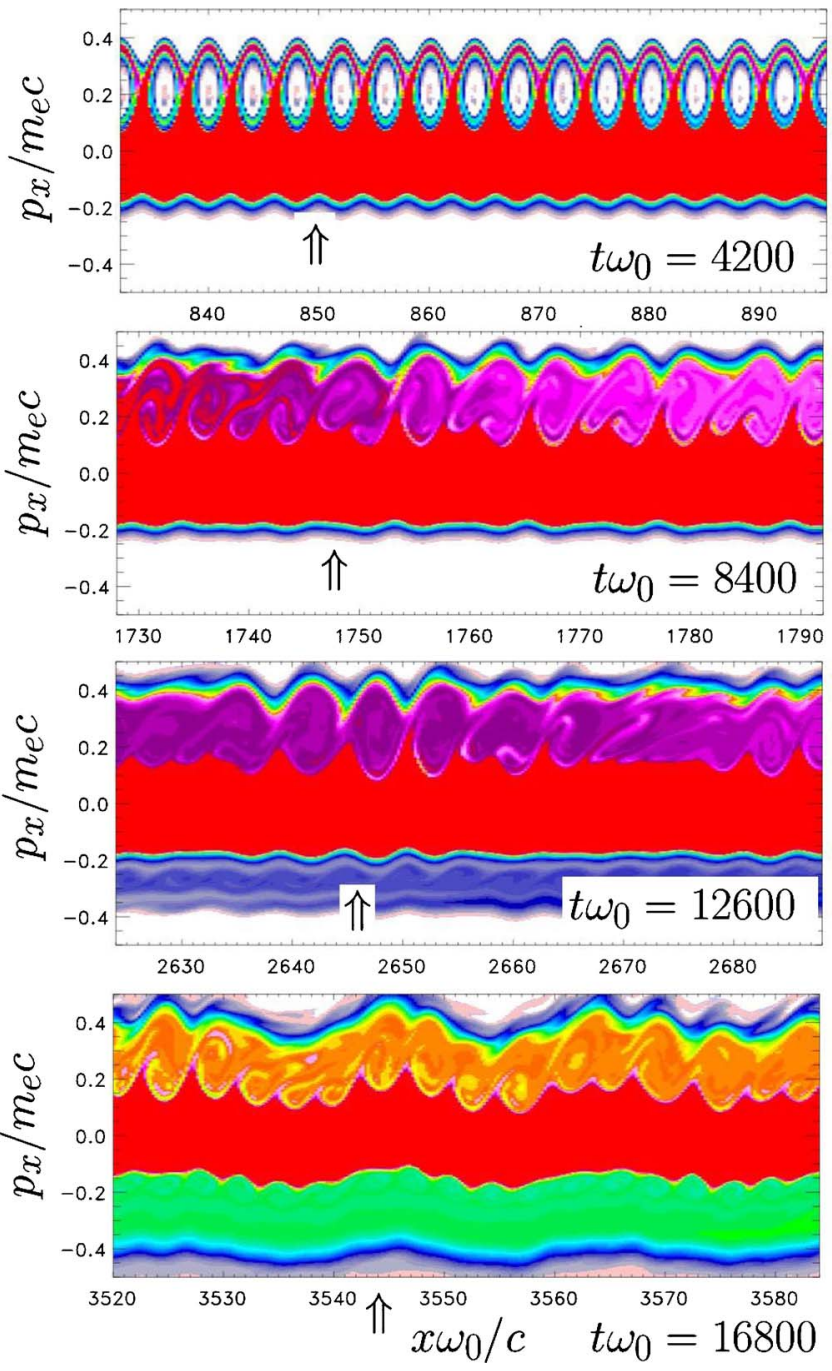

FIG. 8. (Color online) Phase space representation of the electron distribution function in the case of the uniform plasma slab. The top panel $\left(t \omega_{0}\right.$ $=4200$ ) clearly exhibits the trapping and formation of holes (with plasma momentum of 0.218 ) induced by the EPW in the initial state of the instability with a wavevector close to the expected value of $k_{B} c / \omega_{0} \simeq 1.559$. This cell was selected among all the cells at the same time by considering that the wave train motion is made at the linear phase space velocity. We have also added an arrow on the different images indicating the position of a phasespace marker which would be stationary in the frame moving at the (EPW) phase velocity. Somewhat before the time $t \omega_{0}=8400$ (second panel of Fig. 8 ), the system has undergone a transition to a more turbulent regime as reflected in the chaotic electron phase space structure resulting from sequential and (apparently) pairwise coalescence in phase space. The last bottom panel shows the slower spatial modulation induced by SRS-F.

As remarked at the outset of this paper, there is a very interesting set of Vlasov fluid plasma simulation results presented by Brunner and Valeo in Ref. 4 which clearly demonstrate the presence of the Trapped Particle Instability (TPI) and indicate that this temporally correlated the suppression of the SRS scattering and thus the likely cause. In this work we see strong particle kinetic effects also associated with the observed suppression of SRS. However the phenomenon seen here is quite different from that of Brunner and Valeo. Just why this might be so is a subject to which we will return in the conclusions, but just here we will address our reasons for this belief. 
Since we have just presented our data, for ease of comparison we summarize the electrostatic $k$-spectrum data of Brunner and Valeo (=BV hereafter) (i.e., Fig. 1 of Ref. 4) with the dominant component at the SRS-coupled value $k_{B}$. Now, in the classic reduced model of Trapped Particle Instability (TPI) theory as cited in Ref. 4 or more in previous Ref. 23 or Ref. 24, one expects an equal growth (likely exponential) of both the sidebands $\left(k_{B}-\Delta k_{m}, k_{B}+\Delta k_{m}\right)$ and a corresponding decrease in the $k_{B}$ component, giving a trident-like $k$-spectrum, symmetric around $k_{B}$. What seems to have happened in the BV case is some generalization or modification of the basic TPI concept occurred since, although the $k$-values at which the growth occurs are as expected from TPI [as shown in BV's Fig. 5(b) in Ref. 4], the two sidebands grow at very different rates $(3: 1)$, and reach very different levels [both shown in Fig. 5(a) in Ref. 4] the largest being at $k_{B}-\Delta k_{m}$, and the lower being at the upper $k$-component at $k_{B}+\Delta k_{m}$. (Even more impressive is the fine detail of the BV Fig. 2 showing that the modulation moves at the linear group velocity, as one might hope.) Clearly, in the Brunner-Valeo results presented in Ref. 2, some form of the Trapped Particle Instability dominates the behavior of the electrostatic plasma wave system. (Just how this turns off the SRS interaction has yet to be established.)

Returning to our own results, in the light of the TPI $k$-spectra results of Brunner and Valeo in Ref. 4 our main reason for deciding against TPI in our work is the fact that we see a featureless spectrum around $k_{B}$ and another broad feature at $k_{\mathrm{VM}}$ [which is attributed by us to Vortex Merging $(=\mathrm{VM})]$. We see no sign of the TPI modulation spectra (albeit very asymmetric) of the form of three well-separated plasma waves $\left(k_{B}-\Delta k_{m}, k_{B}, k_{B}+\Delta k_{m}\right)$. While it could be speculated that there may have been an early period of TPI and a refinement of our diagnostics might find this, TPI is not a major player in the cases we discuss.

\section{PARABOLIC DENSITY PROFILE}

In considering an application to experimental moderately underdense plasmas, the uniform slab, while excellent for untangling the basic physics, is quite unrealistic. Hence simulations of the same kind as those just discussed were done for an inhomogeneous density profile with the top density corresponding to the homogeneous density $n_{0} \simeq 0.0825 n_{c}$ and a quiver momentum of $a_{0}=0.025$. The system consists of a plasma with parabolic density profile of width $3900 c / \omega_{0}$, peaked at $x_{\mathrm{Max}}=2304 c / \omega_{0}$ surrounded by a vacuum space of $354 c / \omega_{0}$ on both sides. Explicitly, the electron density is $n_{e} / n_{\mathrm{Max}}=1-\left(\left(2304-x \omega_{0} / c\right) / 1950\right)^{2}$, with values greater than zero between $x_{\text {Left }}=354 c / \omega_{0}$ and $x_{\text {Right }}=4254 c / \omega_{0}$. The total length of the system is then $L=4608 \mathrm{c} / \omega_{0}$ corresponding to $702.4 \lambda_{0 \mathrm{vac}}$ (i.e., about $245 \mu \mathrm{m}$ with an incident pump wave of $\lambda_{0}=0.35 \mu \mathrm{m}$ ).

We keep the same electron temperature of $T_{e}=2 \mathrm{keV}$ $=4 T_{i}$ and now ions are taken into account with a mass of $m_{i}=1836 m_{e}$. As for the slab case, the plasma profile, the pump and probe frequencies so that the perfect matching conditions of SRS-B occurs right on the top of the parabolic
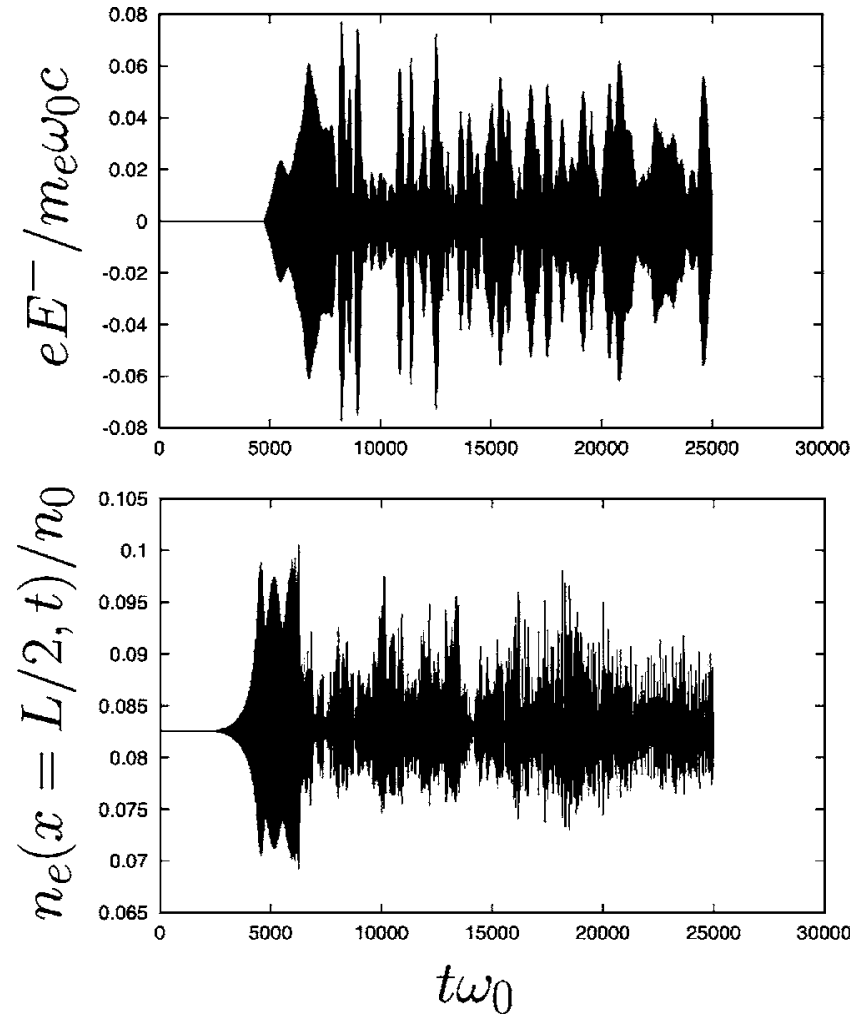

FIG. 9. For the parabolic profile (maximum density of $n_{0} / n_{c}=0.0825$, $a_{0}=0.025$ and $T_{e}=2 \mathrm{keV}$ ), the typical temporal behavior is shown for (a) the scattered mode $e E^{-} / m_{e} \omega_{0} c$ (top panel) and (b) for the density evolution in the middle of the plasma (bottom panel). Two different phases are evident as observed in the uniform slab case (compare Fig. 1): a fluid (coherent) phase at the beginning of the evolution (for $t \omega_{0} \leqslant 7000$ in the plasma, followed by the "burst-like" regime for later times).

profile at a density $n_{\mathrm{Max}}=0.0825 n_{c}$, where both waves (pump and seed) met to start the SRS interaction.

The typical temporal behavior is shown in Fig. 9 for the parabolic profile: the amplified probe $e E^{-} / m_{e} \omega_{0} c$ and the corresponding electron plasma mode $n_{e}\left(x=x_{\operatorname{Max}}, t \omega_{0}\right)$. As for the slab case (see, for instance, Fig. 1), two different phases are evident: a fluid (coherent) phase at the beginning of the evolution (for $t \omega_{0} \leqslant 7000$ in the plasma, and $t \omega_{0} \leqslant 5600$ for the backscattered signal at the left boundary) followed by the "burst-like" regime for later times. We also present in Fig. 10 , at time $t \omega_{0}=25000$ (i.e., at the end of the numerical simulation), the $k$-spectra of the electron density and the backscattered electromagnetic field (probe signal). Note also in Fig. 10 the high level of the Vortex-Merging mode in amplitude in comparison to the SRS-B mode.

For this parabolic density, Fig. 11 shows the normalized backscattered signal $\left|E^{-}(k, \omega)\right|$ spectrum and the corresponding electrostatic $\left|e E_{x}(k, \omega)\right|$ spectrum (to be compared to the slab results of Figs. 4 and 6, respectively). It is clear that similar scattering processes are present involving first modified SRS-B process given by Eq. (7), since there are SRS-B results $\left|e E^{-}(k, \omega) / m_{e} \omega_{0} c\right|$ of Fig. 11 which strongly resemble those of Fig. 4, except that the low-frequency feature $\left(\omega / \omega_{0}=0.45\right.$ and $\left.k c / \omega_{0}=0.37\right)$ of Fig. 4 is no longer evident. There is, however, significant difference in Fig. 11, in that a noticeable amount of unexpected electromagnetic energy 

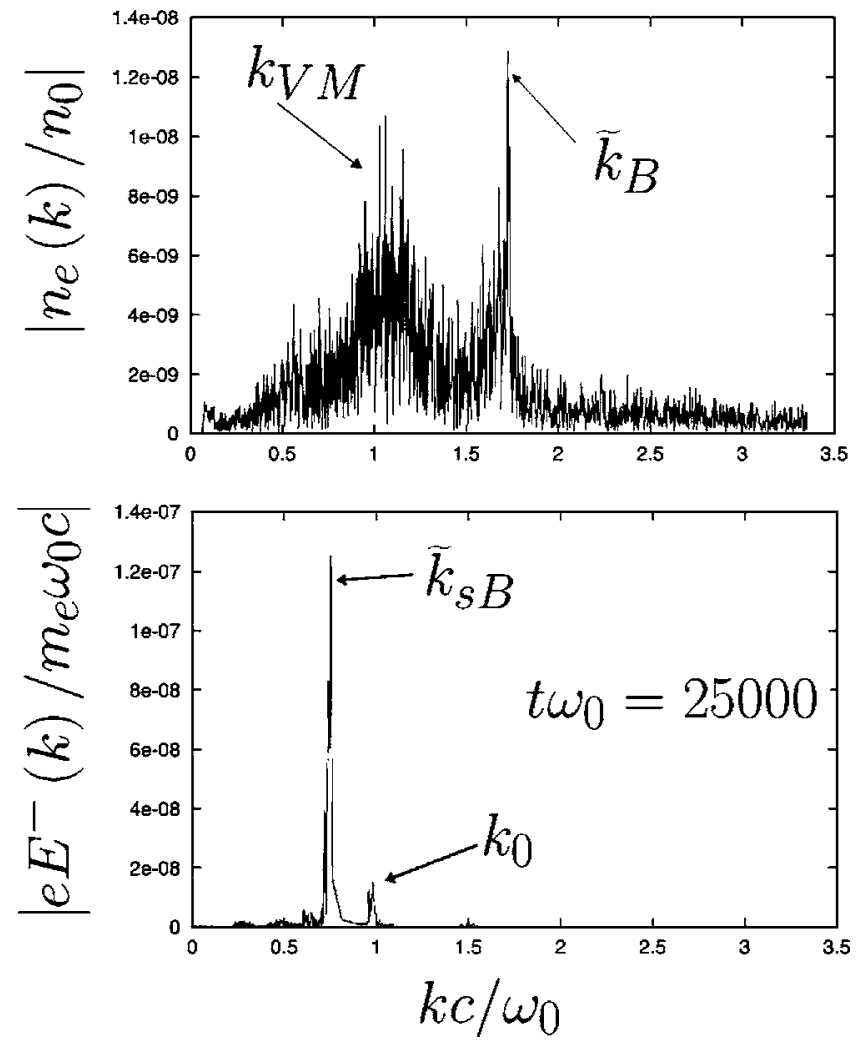

FIG. 10. For the parabolic profile, at time $t \omega_{0}=25000$ (i.e., at the end of the numerical simulation), the $k$-spectra of the electron density (in top panel) and the backscattered electromagnetic field (in bottom panel). Note the somewhat higher level (as compared with Fig. 5 for the slab) of the VortexMerging mode in amplitude in comparison to the SRS-B mode.

$\left|e E^{-}(k, \omega) / m_{e} \omega_{0} c\right|$ which extends down to about the peak plasma frequency. The source of this broadband signal in this "noiseless" Vlasov code is not yet clear. Also absent in Fig. 11 is the equivalent of the SRS-F signal of Fig. 6, probably because of the greater inhomogeneity of the plasma, which is no longer flat topped. Since SRS-F is not excited in the parabolic profile, it is not surprising that the rescatter of Raman forward scatter (SRS-F) by Stimulated Vortex-Merging Scattering (SVMS-B) backscatter is not seen here.

Also, as one might well expect, an inhomogeneous profile leads to a perturbation of the plasma with a larger bandwidth both in frequencies and wavenumbers. The electrostatic spectrum (shown in the bottom panel in Fig. 11) is an example of such behavior. The spectrum reveals a larger broadening with downshifted values of the EPW frequency well below the linear value (corresponding to the BohmGross value at the peak density, plotted in the solid line). This result suggests that weakly damped acoustic-like modes were excited inside the plasma at densities below the peak density. The behavior of the electron distribution function (see, for instance, Fig. 13 or Fig. 14) and in particular the vortex merging leads to structures similar to BGK waves. In Ref. 15 these resulting modes were called by Vu et al. BAM modes for Beam Acoustic Modes.

Further information on these physical processes in the parabolic profile is available in Fig. 12 (to be compared with the equivalent results of Fig. 7 for the slab plasma) in the
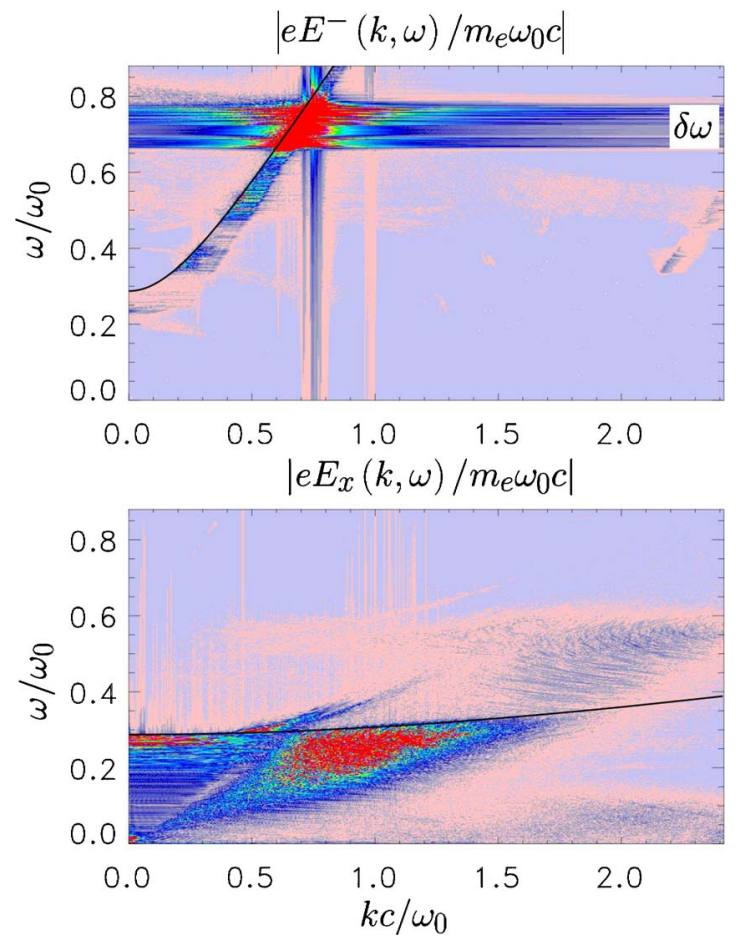

FIG. 11. (Color online) Backscattered field spectrum in the plane $(k, \omega)$ (top panel) and the corresponding electrostatic field spectrum in the bottom panel in the case of the parabolic profile. The inhomogeneous profile leads to a perturbation of the plasma with a larger bandwidth both in frequencies and wavenumbers. The electrostatic spectrum (shown in the bottom panel) is an example of such a behavior. The spectrum reveals a larger broadening with downshifted values of the EPW frequency well below the linear value (corresponding to the Bohm-Gross value plotted in solid line). This result suggests that weakly damped acoustic-like modes were excited inside the plasma.

form of the $\left|e E_{y}(k, t) / m_{e} \omega_{0} c\right|$ and $\left|n_{e}(k, t) / n_{0}\right|$. The shift in electromagnetic scattered wavevector leads to a value of $\widetilde{\omega}_{s B} \simeq 0.765 \omega_{0}$ for the backscattered wave and to the downshifted value of $\widetilde{\omega}_{B} \simeq 0.235 \omega_{0}$ for $\mathrm{EPW}$.

From the backscattered spectrum, Stimulated Brillouin Back-Scattering (SBS-B) seems to play a minor role in this (kinetic) regime, at least for this set of physical parameters. It is important to keep in mind that from the initial SRS-B matching relations that we recall now, $\omega_{0}=\omega_{s B}+\omega_{B}$ and $k_{0}=-k_{s B}+k_{B}$, we have slightly moved through the nonlinear shift to a new set of conditions $\omega_{0}=\widetilde{\omega}_{s B}+\widetilde{\omega}_{B}\left(\omega_{0}=0.765 \omega_{0}\right.$ $\left.+0.235 \omega_{0}\right) \quad$ and $k_{0}=-\widetilde{k}_{s B}+\widetilde{k}_{B} \quad\left(0.957 \omega_{0} / c=-0.710 \omega_{0} / c\right.$ $\left.+1.667 \omega_{0} / c\right)$ in which the EPW is no more a Langmuir wave obeying the linear dispersion relation. The time evolution of the $k$-spectrum of the electromagnetic field $e E_{y} / m_{e} \omega_{0} c$ and of the electron density shown respectively in the top panel and the bottom panel of the Fig. 12 show a change that is consistent with the combined result of the nonlinear frequency shift due to trapping effects and the continuation of the shifted SRS-B resonance. In the upper panel, the change of $k_{s B}$ to upshifted values begins a little earlier than for the slab, and ceases around the time $t \omega_{0}=11000$, indicating a saturation of the chirp. The inverse cascade characterized at the outset by the pairwise vortex coalescence is visible in the $\left|n_{e}(k, t) / n_{0}\right|$ data; it is much wider than for the slab case of Fig. 7. 

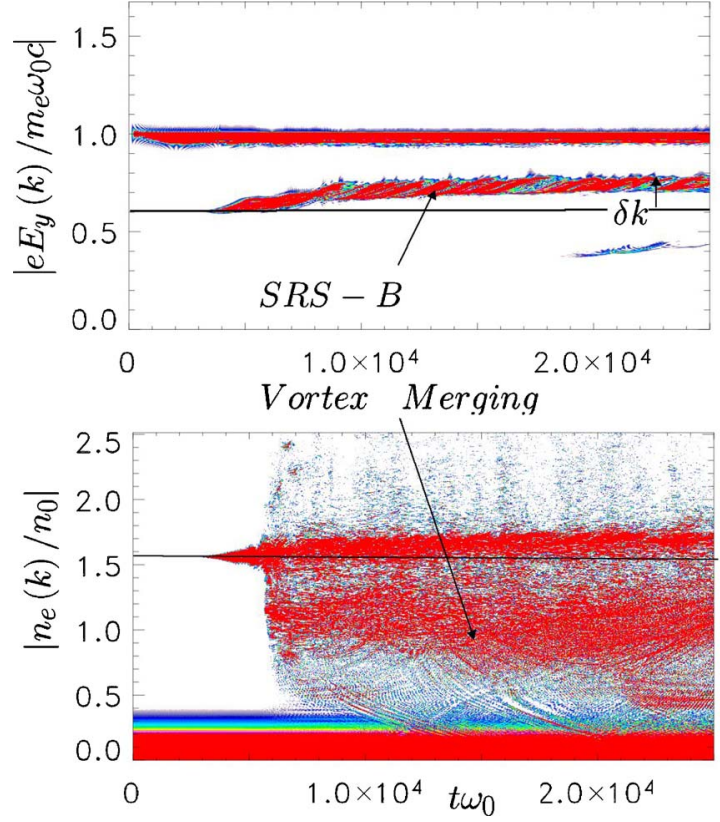

FIG. 12. (Color online) For the parabolic plasma, the time evolution of the $k$-spectrum of the electromagnetic field $\left|e E_{y}(k, t) / m_{e} \omega_{0} c\right|$ and of the electron density $\left|n_{e}(k, t) / n_{0}\right|$ are shown, respectively, in the top panel and the bottom panel. We observe a change that is consistent with the combined result of the nonlinear frequency shift due to trapping effects and the maintenance of the SRS-B resonance. The process leading to lower wavenumbers ("inverse cascade") beginning with pairwise vortex coalescence is clearly visible in the bottom panel and is much broader in $k$ than the corresponding VM process for the slab as shown in the bottom panel of Fig. 7.

\section{PHASE SPACE REPRESENTATION AND EVIDENCE OF VORTEX MERGING}

In the Vlasov simulations discussed here, the main process that limits the Langmuir wave three-wave resonant frequency-wavevector chirping of SRS-B in its "kinetic" form (i.e., when LDI does not dominate) is provided by the process which begins with pairwise merging of phase space holes and was here labeled "inverse cascade" (in which there is a continuous flow toward lower wavenumbers and lower frequencies, although, perhaps, "downward chirp" might be more appropriate). This is the process which is seen to break up the chirped resonance tracking in the kinetic regime, after which dramatic electrostatic wavenumber lowering and broadening is apparent. We now address here the evidence for this process that ultimately limits the scattering of the injected pump electromagnetic wave.

The structures resulting from SRS-B instability growth are characterized by the formation of strong trapping vortices have in effect modified the plasma enough so that the linear Landau damping has been destroyed. After these vortices are well formed the waves are found to be very stable and selfsustained. (In other simulations they indeed persist over a long time after the pump was turned off.) These waves were first generated as classic Langmuir waves plasma waves associated with SRS-B, with enough energy invested to overcome the considerable linear Landau damping and with normalized frequency and wavenumber here nonlinearly lowered by Morales and O'Neil retuning to become Langmuir-Morales-O'Neil (LMO) waves with normalized frequency and wavevector of $\widetilde{\omega}_{B}=\omega_{B}-\delta \omega \geqslant 0.25 \omega_{0}$ and $\widetilde{k}_{B}$ $=k_{B}+\delta k \leqq 1.65 \omega_{0} / c$. [It may well be the case that enough drive to obtain well-organized phase-space vortices in this kinetic regime $\left(k_{B} \lambda_{\mathrm{De}} \geqslant 0.3\right)$ will automatically require significant LMO retuning, i.e., the two will go hand-in-hand.]

Rather than continuing this retuning three-wave resonance process indefinitely, these coherent and three-waveresonant LMO waves for some reason began a phase space pairwise vortex merging process at well-separated (but apparently uncorrelated) sites in the moving wave-train, a process which broke up the three-wave resonance. While something like this vortex merging was observed by Bertrand et al. in Ref. 22 in their purely periodic pure two-beam initial-value simulation, in that case the merging proceeded until only one vortex was left in the simulation box; the merging went as far as it could go. That is not what happened here. The spatial Fourier transform analysis here in this large simulation showed that the average wavenumber was reduced by the modest factor of 0.65 (rather the 0.5 limit one might expect if each adjacent pair of vortices had merged). While the initial temptation is to look at the phase velocity and the spatial structure to infer a frequency, the spectra of Fig. 6 and Fig. 11, the apparent phase velocity changes little so this would impose a proportionality on the behavior of $\omega$ and of $k$. However the observed behavior indicates much more change in wavenumber $k$ than in frequency $\omega$. The answer to this apparent paradox is that once the phase space resonance vortices are considerably melded, the behavior becomes that of more or less uncorrelated plasma waves and their behavior is that of the dispersion of the Langmuir wave but depressed by the Morales-O'Neil nonlinear frequency change. In other words, once the vortices cease to be wellconnected, the behavior becomes that of a plasma with a modified distribution of fast electrons as discussed by Yin et al. in Refs. 16 and 17 and by Strozzi et al. in Ref. 14.

Such a phenomenon or the vortex-merging tendency has been recently observed by Valentini, O'Neil, and Dubin in Ref. 25 in the case of the study of (Trapped) Electron Acoustic Waves (TEAWs) in the nonlinear regime using periodic and electrostatic PIC simulations. These authors have investigated the possibility of decay of these TEAWs to longer wavelengths which appears as a tendency of the vortex-like trapped particle populations to merge. Indeed these modes are characterized by phase space holes and are similar to BGK equilibria. When this TEAW is replicated in space and used as an initial condition for the simulation, the wave is observed to decay to longer wavelength TEAW and then to shorter wavenumbers. Such modes behave as BGK modes and previous theory in Refs. 26 or 27 have suggested that BGK modes may also subject to such decay instabilities.

In Fig. 13 the phase space plots of a given region of the plasma are presented at various times during the time evolution of the plasma, being the distribution function $f\left(x, p_{x}\right)$ at the times $t \omega_{0}=5600,7000,8800$, and 12400 for the second simulation case corresponding to a parabolic profile. The electrons are trapped and accelerated with positive velocities in the vicinity of the phase velocity of the $\mathrm{EPW} \quad v_{\phi}(\mathrm{EPW})=\omega_{B} / k_{B}=0.3333 / 1.5594=3.41 v_{\text {th }}$ or 

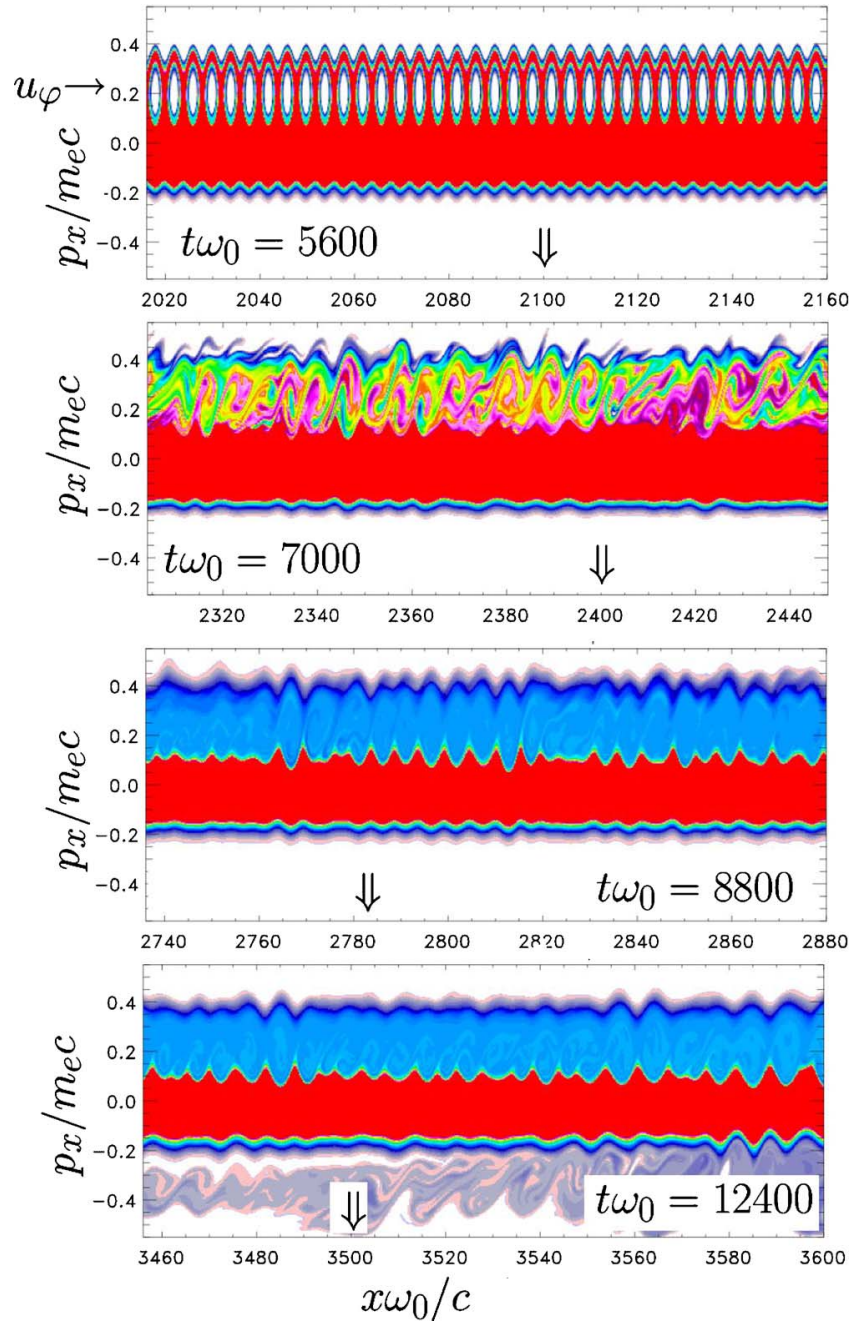

FIG. 13. (Color online) For the parabolic profile (centered at $x \omega_{0} / c=2304$, zero-density edges at $\left.x \omega_{0} / c=354,4254\right)$, the phase space plots of the electron distribution function beginning near the center are shown with the subsequent frames following at about the electrostatic plasma wave (EPW) phase velocity. The first image clearly exhibits the formation of very neat phase space hole wave train, after the particle trapping of been completed in the EPW induced by SRS-B. The second snapshot at $t \omega_{0}=7000$ is made by placing the window (of width $\Delta x \omega_{0} / c=140$ ) as predicted by the SRS wavematched phase velocity. [The arrow on the different frames indicates the position of the corresponding vortex which is stationary in the frame moving at the (EPW) phase velocity.] After the first frame the EPW begins the bursting or turbulent regime. In the second frame considerable vortex merging has taken place and phase space fine structure in the form of "tendrils" is clearly evident. (In the last frame at the bottom of the distribution, one finally begins to see accelerated electrons which were reflected in the ambipolar sheath field and are passing the EPW region but now moving leftward towards the laser. The fact that this arrives so late emphasizes the fact that the evolution of the instability has nothing to do with recently heated electrons producing strong damping, the mechanism commonly seen in smaller simulations.)

$u_{\phi}(\mathrm{EPW})=p_{\phi} / m_{e} c=0.218$, and we follow the trapping structures or phase space holes during their motion in the phase space. The $x$ space (for the total length of the system) has been divided in 32 cells, with each cell having a length of $144 c / \omega_{0}$. The first cell, shown at time $t \omega_{0}=5600$, in the top panel in Fig. 13, was initially chosen in front of the top of the parabolic profile in the region of optimum interaction of both electromagnetic waves (pump and seed). There are 36 vorti-
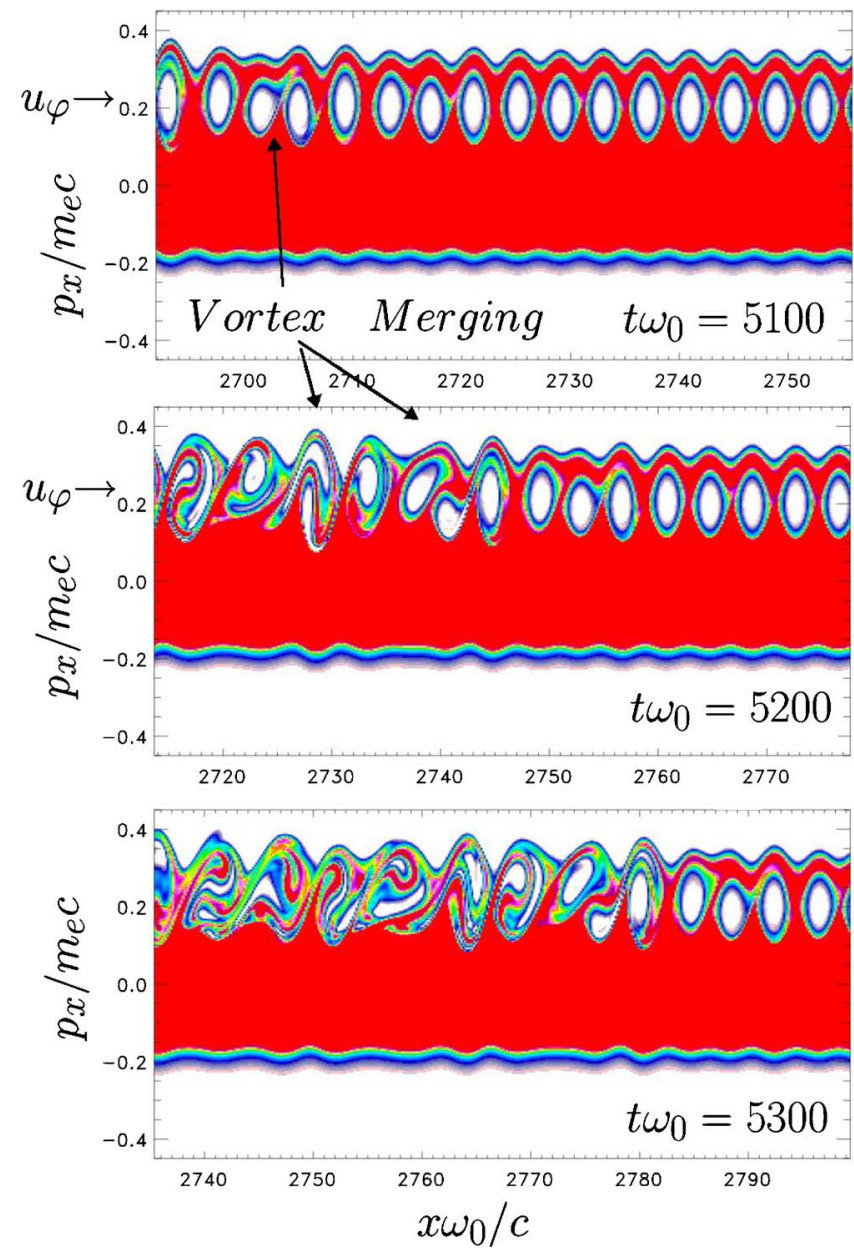

FIG. 14. (Color online) In the parabolic-profile plasma, some phase space snapshots, still moving at the resonance phase velocity, but at shorter intervals than in earlier figures $\left(\Delta t \omega_{0}=100\right)$ are presented to show the details of the early development of the vortex merger process. [This second simulation was carried out keeping ions fixed and with a somewhat larger quiver momentum of $a_{0}=0.030$ (as compared with 0.025 of the earlier figures).] There are about $16+$ vortices in the window and the coalescence begins with the symmetry-breaking of an apparently random pair of vortices, and then spreads out to involve more and more neighboring vortices. It is important to note that this case is not one with a uniform periodic plasma evolving from an unstable initial state (as in the very two-stream first vortex merger work of Bertrand et al. in Refs. 22 and 26), but for a much more realistic case. Although phase space vortex merging does begin with symmetrybreaking in space, this work shows that this phenomenon is not critically dependent on overall spatial uniformity and occurs as well in more realistic density profiles.

ces in a plasma sample of length of $144 c / \omega_{0}$, leading to a wavevector of $k_{B} c / \omega_{0} \simeq 1.57$ in good agreement with the linear expected value of $k_{B} c / \omega_{0}=1.559$. The space-momentum distribution function clearly exhibits the formation of wellformed phase space holes after trapping of particles. The arrow marking the tagged point at $x \omega_{0} / c=2100$ is at that point somewhat to the left of the density peak at $x \omega_{0} / c$ $=2304$.

The second plot at time $t \omega_{0}=7000$ is obtained by following the vortex motion in phase space. At this time one can see clearly that the behavior of this region of phase space is now much more turbulent, and much more turbulent than what is shown in Fig. 3 of Brunner and Valeo in Ref. 4. The arrow marking the tagged point is now at $x \omega_{0} / c=2400$ is at 
$x \omega_{0} / c=2400$ that point somewhat to the right of the density peak at $x \omega_{0} / c=2304$.

At time $t \omega_{0}=8800$ the cell exhibits now about 29 trapping structures giving an average wavenumber which has dropped to a value of $k_{\mathrm{VM}^{c}} / \omega_{0} \simeq 1.26$. At time $t \omega_{0}=12400$, the cell arrow is now located at $x \omega_{0} / c=3500$ in the region of slightly lower density $(1-0.0603)$ from the peak for the plasma [so the linear wavenumber would have increased slightly by only $3 \%$ to $\left.k_{B}(x) c / \omega_{0} \simeq 1.62\right]$. However no such structure is visible and one sees 26 trapping structures corresponding to an average value of the wavevector of $k_{\mathrm{VM}} c / \omega_{0} \simeq 1.13$.

The ratio of the phase velocity to the electron thermal velocity $\left(v_{\phi} / v_{\text {th }}\right)$ is then easily and accurately measured at time $t \omega_{0}=8800$ as $v_{\phi}(\mathrm{VM})=\omega_{\mathrm{VM}} / k_{\mathrm{VM}} \simeq 0.235 / 1.26$ $\simeq 2.98 v_{\text {th }}$ (i.e., $u_{\phi}=p_{\phi} / m_{e} c \simeq 0.19$ ) while for the EPW modified by trapping induced nonlinear shift, we have $v_{\phi}(\mathrm{NLEPW}) / c=\widetilde{\omega}_{B} / \widetilde{k}_{B} c \simeq 0.235 / 1.667$, or $\widetilde{\omega}_{B} / \widetilde{k}_{B} \simeq 2.25 v_{\text {th }}$ or $u_{\phi}(\mathrm{NLEPW})=0.142 c$ (for the nonlinear Electron Plasma Wave) to compare to the linear value of $v_{\phi}(\mathrm{EPW})=\omega_{B} / k_{B}$ $=0.3333 c / 1.5594=3.41 v_{\text {th }}$ or $u_{\phi}(\mathrm{EPW})=0.218 c$.

As a consistency check, the origin of this electrostatic turbulence can be identified by considering a window of length $62.5 \mathrm{c} / \omega_{0}$, moving at the linear phase velocity of the EPW created by SRS-B $v_{\phi}(\mathrm{EPW})=\omega_{B} / k_{B}$. A second simulation (with identical physical parameters but keeping ions fixed) has been carried out with $a_{0}=0.03$. Since the window is carefully tailored to move at the phase velocity $v_{\phi}$ of the EPW, the holes are now quite stationary in this frame (at least for the beginning of the simulation when the nonlinear shift induced by trapping effects is not too high), giving a time history of the same population of trapped particles.

To show in more detail how the initial phase-space vortex merging proceeds, we show in Figs. 14 and 15 the detailed history over a fairly short time $\left(t \omega_{0}=5100,5200\right.$, and 5300 in Fig. 14) of how the vortices on the window moving at the wave velocity begin to coalesce and lose their coherence although still in the presence of significant fields whose waveform is becoming noticeably nonsinusoidal. The early pairwise vortex merging phenomenon is located in the first frame at the left side of the box near the position $x \omega_{0} / c$ $=2700$. We are located well away from the top of the parabolic profile towards the right side of the plasma. Immediately after this in Fig. 15 the behavior is shown of this same particle population as the time increases. The top panel at $t \omega_{0}=5400$ shows a typical result when merging is well advanced and while considerable structure is still seen, the mixing is well advanced. At the next time frame, $t \omega_{0}$ $=5500$, the reformed phase space structure as a result of the coalescence have now a wavevector close to $k_{\mathrm{VM}} c / \omega_{0}$ $\simeq 1.10$. The bottom panel in Fig. 15 shows the asymptotic behavior at time $t \omega_{0}=5900$ showing that such trapping structures may persist over a long time even in regions of where the electron density has become fairly well mixed on a scale comparable to the average wavelength of the plasma disturbance. Close inspection shows that there is considerable fine structure still remaining.

These sequences show clearly how quickly the plasma

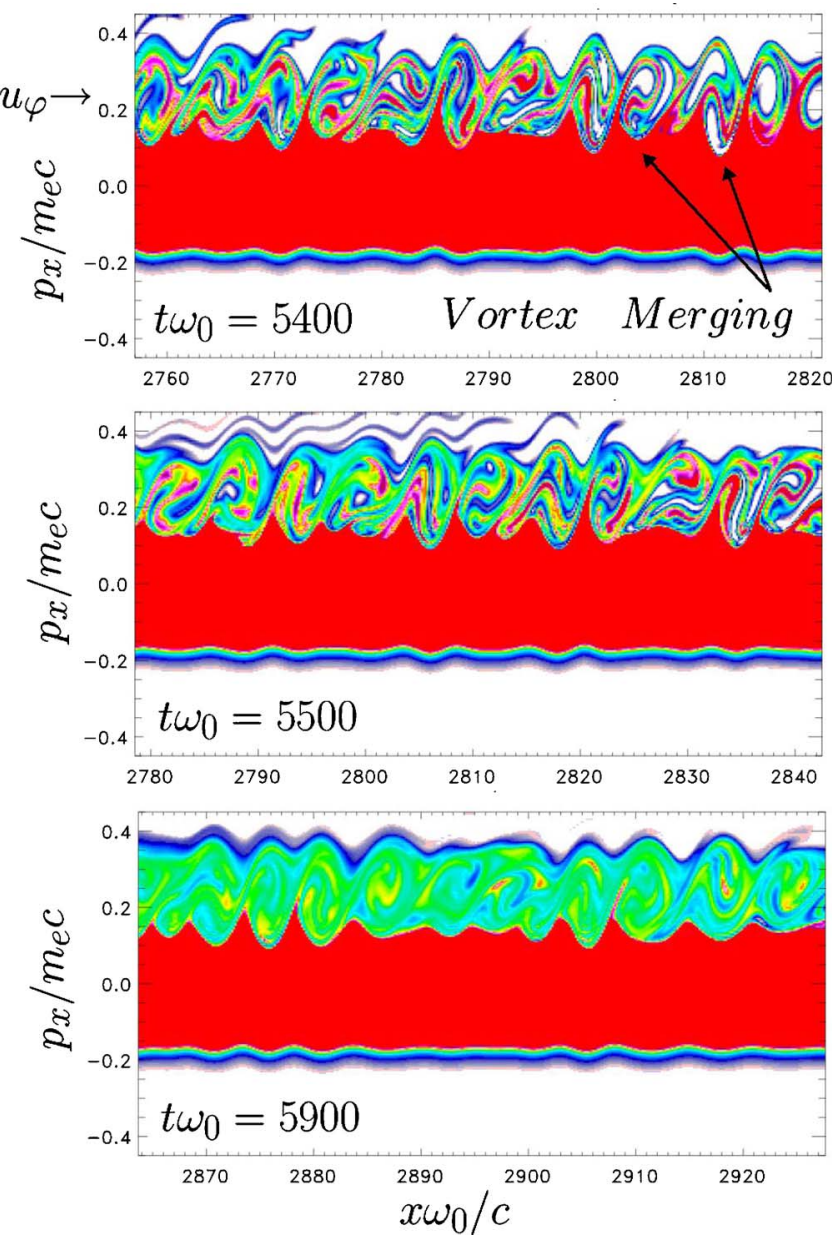

FIG. 15. (Color online) Continuing on from the previous set of phase space frames in Fig. 14, following the vortices, one can see how the spatial frequency of the waveform is being reduced in a fairly stochastic way and how the distribution of trapped electrons, although incompressible in phase space, are being considerably mixed in phase space. Although the phase space structure is being reformed into a somewhat coarser lowerwavenumber configuration, the electron motion is still quasitrapped (even though the waveform is no longer quasistationary in the moving frame) and there is no evidence for simple convective shear in phase space. Rather than remaining almost locked in the original form, it seems as if the system now behaves more like an ensemble of nonlinear EPW and more or less satisfies the Langmuir dispersion relation. It is likely that if this distribution function were to be space-averaged and analyzed, the Ballistic Acoustic Modes and Beam modes discussed by Vu et al. (Ref. 15), Yin et al. (Refs. 16 and 17), and Strozzi et al. (Ref. 14) would emerge.

moves from a classic fully trapped well-organized plasma wave to a structure with much more phase-space mixing and incoherence.

\section{CONCLUSION}

In summary, the SRS-B instability was numerically investigated in two large plasmas using a semi-Lagrangian Vlasov model. The observed behavior corresponds to the combined action of the frequency shift of the amplified EPW induced by nonlinear trapping effect (first analyzed by Morales and O'Neil), compensated for by a retuning of the wavenumber as well, so as to maintain the parametric resonance over a long time. The simulations show that the main process that limits the continuation of the SRS-B instability 
by Langmuir wave frequency-wavevector chirping and retuning is provided by a process which begins with pairwise vortex merging of phase space vortices or "holes." The vortex merging of EPWs of shifted frequencies leads to the formation of lower-wavenumber BGK-type structures whose the self-sustained character allows them to persist over a long time.

At the conclusion of the discussion on our slab results (end of Sec. IV) we discussed our Vortex-Merging (VM) SRS results in comparison with the very interesting Trapped Particle Instability (TPI) SRS results of Brunner and Valeo presented in Ref. 4 (BV). The discussion there was confined to making plain the difference between the two types of results, and the discussion of why such difference results was deferred until later. Here we discuss likely reasons for this difference. Since the Brunner-Valeo (BV) plasma is uniform, it is best compared with the slab simulation in this paper. Both simulations are large, the BV distance between the antennas is $4000 \lambda_{\text {De }}$, while our slab is a little under $18000 \lambda_{\text {De }}$; clearly both are large uniform plasma slabs many Debye lengths in size. The temperatures in the two simulations are comparable (the $\mathrm{BV} T_{e}$ is $1.5 \mathrm{keV}$, ours is $2.0 \mathrm{keV}$ ). The main difference between the two simulations lies in the plasma densities chosen. In the BV case the density is $n_{0} / n_{c}=(1 / 2.065)^{2}=0.2345$, while ours is noticeably lower, being $n_{0} / n_{c}=0.0825$. The important result of this difference is the significant difference in the difference in the scattering wavevectors $\left(k_{s} \lambda_{\mathrm{De}}\right)$ values, which are 0.26 for $\mathrm{BV}$ and 0.34 for our work. The phase velocities of these waves in terms of electron thermal velocity $v_{\text {th }}$ were thus quite different, being $\omega_{B} / k_{B} v_{\text {th }}=4.2$ for BV (and thus with relatively weak initial Landau damping), while our value was somewhat less, being $\omega_{B} / k_{B} v_{\text {th }}=0.214 c / v_{\text {th }}=3.42$. (The figure of $0.214 \mathrm{c}$ is given near the end of Sec. V.) This apparently modest change makes a large difference in the initial Landau damping (and thus in the onset of the LDI and TPI phenomena).

Now, our $k_{B} \lambda_{\mathrm{De}}$ value was expressly chosen so as to address the "kinetic" regime $\left(k_{B} \lambda_{\mathrm{De}} \sim 0.32\right)$ where LDI (Langmuir Decay Instability was no longer effective), while $\mathrm{BV}$ value was evidently chosen to be in what has been considered the LDI regime, i.e., for $k_{B} \lambda_{\mathrm{De}} \leqslant 0.28$ or so. Even with an LDI favoring value for $k_{B} \lambda_{\text {De }}$, since LDI is not a very strong process, it was always subject to the implicit caveat "absent any more rapid instability," among which the Modulational Instability (MI) had been a serious candidate. What was particularly striking in the BV result was the unprecedented appearance of TPI apparently dominating LDI (rather than MI). (The previous context for TPI had been the electron beam excitation of plasma waves.)

The question which remains is to understand when one might expect TPI, as in the BV simulation, and when VM (Vortex Merging) as in our work. In view of this discussion, the most plausible explanation is that one will pass from one to the other as $k_{B} \lambda_{\mathrm{De}}$ increases from 0.26 (for BV's TPI) and 0.34 (for our VM). However, having had to devote so much space to establish VM in this work, it seems that the task of studying such a transition from one regime to the other must await a future publication. For this publication it must suffice to content ourselves with these few lines presenting the plau- sible speculation of dependence of the behavior on the value of $k_{B} \lambda_{\text {De }}$. Another topic to be left for future work is how these possibilities are transmuted in a parabolic density profile which is likely to be a more realistic case. There is no doubt much to be done here.

The work presented here has been done only on the first transition from crisply resonant SRS-B behavior to the "bursty" behavior and modification of the electrostatic waves in the plasma. Still to be investigated is the nature of the later bursts. Clearly these a more complicated and costly simulations, but it is these long-term conditions which may well dominate any experiment on underdense plasmas except those with extremely short pulse lengths. In future work to improve our understanding of the SRS-B it will probably be necessary to apply more sophisticated diagnostics for the available signals in the code. The fine-scale gray density time-space diagnostics of Brunner and Valeo in Ref. 4 are likely to play a central role, while for frequency diagnostics quasiresolved in time, techniques such as those treated by Patrick Flandrin (see, for instance, Ref. 28) should be considered.

\section{ACKNOWLEDGMENT}

The authors acknowledge the IDRIS center for computer time allocation on the IBM-Power4 computer.

${ }^{1}$ L. J. Suter, S. Glenzer, S. Haan, B. hammel, K. Manes, N. Meezan, J. Moody, M. Spaeth, K. Oades, and M. Stevenan, Nucl. Fusion 44, S140 (2004).

${ }^{2}$ J. Tassart, Nucl. Fusion 44, S134 (2004).

${ }^{3}$ H. X. Vu, D. F. Dubois, and B. Bezzerides, Phys. Rev. Lett. 86, 4306 (2001); Phys. Plasmas 9, 1745 (2002).

${ }^{4}$ S. Brunner and E. J. Valeo, Phys. Rev. Lett. 93, 145003 (2004).

${ }^{5}$ J. L. Kline, D. S. Montgomery, B. Bezzerides, J. A. Cobble, D. F. Dubois, R. P. Johnson, H. A. Rose, L. Yin, and H. X. Vu, Phys. Rev. Lett. 94, 175003 (2005)

${ }^{6}$ D. S. Montgomery, R. J. Focia, H. A. Rose, D. A. Russel, J. A. Cobble, J. C. Fernandez, and R. P. Johnson, Phys. Rev. Lett. 87, 155001 (2001); Phys. Plasmas 5, 2311 (2002).

${ }^{7}$ V. B. Krapchev, Phys. Rev. Lett. 42, 497 (1979).

${ }^{8}$ A. K. Ram, Phys. Rev. A 22, 1229 (1980).

${ }^{9}$ J. P. Halloway and J. J. Dorning, Phys. Rev. A 44, 3856 (1991); C. Lancellotti and J. J. Dorning, J. Math. Phys. 40, 3895 (1999).

${ }^{10}$ I. B. Bernstein, J. M. Greene, and M. D. Kruskal, Phys. Rev. 108, 507 (1957).

${ }^{11}$ B. B. Afeyan, K. Won, V. Savchenko, T. W. Johnston, A. Ghizzo, P. Bertrand, Third International Conference Inertial Fusion Sciences and Applications M034, Monterey, California, September 7-12, 2003.

${ }^{12}$ L. Nikolic, M. M. Skoric, S. Ishiguro, and T. Sato, Phys. Rev. E 66, 036404 (2002)

${ }^{13}$ A. Ghizzo, T. W. Johnston, T. Reveille, P. Bertrand, and M. AlbrechtMarc, Phys. Rev. E 74, 046407 (2006).

${ }^{14}$ D. J. Strozzi, E. A. Williams, A. B. Langdon, and A. Bers, Phys. Plasmas 14, 013104 (2007).

${ }^{15}$ H. X. Vu, L. Yin, D. F. DuBois, B. Bezzerides, and E. S. Dodd, Phys. Rev. Lett. 95, 245003 (2005).

${ }^{16}$ L. Yin, B. J. Albright, B. Bezzerides, D. F. DuBois, J. M. Kindel, and H. X. Vu, Phys. Rev. E 73, 025401(R) (2006).

${ }^{17}$ L. Yin, W. Daughton, B. J. Albright, K. J. Bowers, D. S. Montgomery, J. L. Kline, J. C. Fernandez, and Q. Roper, Phys. Plasmas 13, 072701 (2006).

${ }^{18}$ D. W. Forslund, J. M. Kindel, and E. L. Lindman, Phys. Fluids 18, 1017 (1975).

${ }^{19}$ S. C. Wilks, W. L. Kruer, K. Estabrook, and A. B. Langdon, Phys. Fluids B B4, 2794 (1992); see also K. Estabrook, W. L. Kruer, and E. A. Williams, Phys. Fluids 31, 372 (1988); K. Estabrook and W. L. Kruer, ibid. 26, 1892 (1983). 
${ }^{20}$ G. J. Morales and T. M. O’Neil, Phys. Rev. Lett. 28, 417 (1972); A. Lee and G. Pocobelli, Phys. Fluids 15, 2351 (1972).

${ }^{21}$ A. Ghizzo, P. Bertrand, M. Shoucri, T. W. Johnston, E. Fijalkow, and M. R. Feix, J. Comput. Phys. 90, 431 (1990); T. W. Johnston, P. Bertrand, A. Ghizzo, M. M. Shoucri, E. Fijalkow, and M. R. Feix, Phys. Fluids B 4, 2523 (1992); P. Bertrand, A. Ghizzo, S. J. Karttunen, T. J. H. Pattikangas, R. R. E. Salomaa, and M. Shoucri, Phys. Plasmas 2, 3115 (1995); A. Ghizzo, P. Bertrand, J. Lebas, T. W. Johnston, and M. Shoucri, ibid. 3, 650 (1996); 5, 4041 (1998).

${ }^{22}$ P. Bertrand, A. Ghizzo, M. R. Feix, E. Fijalkow, P. Mineau, N. D. Suh, M. Shoucri, Proceedings of the International Workshop on Nonlinear Phenomena in Vlasov Plasmas, Cargèse, France, 11-16 July 1988, edited by
F. Doveil, Editions de Physique d'Orsay, p. 109 (1989).

${ }^{23}$ W. L. Kruer, J. M. Dawson, and R. N. Sudan, Phys. Rev. Lett. 23, 823 (1969).

${ }^{24}$ D. A. Hartmann and C. F. Driscoll, Phys. Plasmas 8, 3457 (2001).

${ }^{25}$ F. Valentini, T. M. O'Neil, and D. H. E. Dubin, Phys. Plasmas 13, 052303 (2006).

${ }^{26}$ A. Ghizzo, B. Izrar, P. Bertrand, E. Fijalkow, M. R. Feix, and M. M. Shoucri, Phys. Fluids 31, 72 (1988).

${ }^{27}$ H. L. Berk, C. E. Nielsen, and K. V. Roberts, Phys. Fluids 13, 980 (1970).

${ }^{28}$ Patrick Flandrin, Time-Frequency/Time-Scale Analysis (Academic, New York, 1998). 